\title{
Strong entanglement distribution of quantum networks
}

\author{
Xue Yang $\odot,{ }^{1}$ Yan-Han Yang $\odot,{ }^{1}$ and Ming-Xing Luo ${ }^{1,2, *}$ \\ ${ }^{1}$ The School of Information Science and Technology, Southwest Jiaotong University, Chengdu 610031, People's Republic of China \\ ${ }^{2}$ Shenzhen Institute for Quantum Science and Engineering, Southern University of Science and Technology, \\ Shenzhen 518055, People's Republic of China
}

(Received 7 October 2021; accepted 11 February 2022; published 24 February 2022)

\begin{abstract}
Large-scale quantum networks have been employed to overcome practical constraints of transmission and storage for single entangled systems. Our goal in this paper is to explore the strong entanglement distribution of quantum networks. We firstly show that any connected network consisting of generalized Einstein-PodolskyRosen states and Greenberger-Horne-Zeilinger states satisfies the strong Coffman-Kundu-Wootters monogamy inequality in terms of the bipartite entanglement measure; in addition, the monogamy inequalities are also considered for generic entangled quantum networks. This reveals the interesting feature of high-dimensional entanglement with local tensor decomposition going beyond qubit entanglement. We then apply the entanglement distribution relation in entangled networks to get a quantum maximum-flow minimum-cut theorem in terms of von Neumann entropy and Rényi- $\alpha$ entropy. We finally classify entangled quantum networks by distinguishing network configurations under local unitary operations. These results provide insights into characterizing quantum networks in quantum information processing.
\end{abstract}

DOI: 10.1103/PhysRevResearch.4.013153

\section{INTRODUCTION}

Entanglement as an intriguing phenomenon has been considered to be the heart of quantum mechanics. It provides a crucial resource for quantum information processing, including quantum teleportation [1], quantum dense coding [2], quantum secret sharing [3], and quantum cryptography [4]. The study of entanglement and its distribution reveals fundamental insights into the nature of quantum correlations $[5,6]$, the features of many-body systems $[7,8]$, and the potential limitations for quantum-enhanced technologies [9].

One interesting feature of entanglement is the impossibility of sharing entanglement freely in multiparty quantum systems. This, without any classical counterpart, is known as the monogamy of entanglement (MOE) [10,11]; that is, entanglements satisfy special constraints on how they can be distributed among multipartite systems. The monogamy inequality became synonymous due to Coffman, Kundu, and Wootters (CKW) [11] with

$$
\mathcal{Q}_{\mathrm{A} \mid \mathrm{BC}}\left(\rho_{\mathrm{ABC}}\right) \geqslant \mathcal{Q}_{\mathrm{A} \mid \mathrm{B}}\left(\rho_{\mathrm{AB}}\right)+\mathcal{Q}_{\mathrm{A} \mid \mathrm{C}}\left(\rho_{\mathrm{AC}}\right),
$$

with specific entanglement measure $\mathcal{Q}$. Here, $\rho_{\mathrm{AB}}=$ $\operatorname{Tr}_{C}\left(\rho_{A B C}\right)$ denotes the reduced state of parties $A$ and $B$, and analogously for $\rho_{A C}$. A $\mid B C$ is for the bipartite split. The first example is related to the squared concurrence between bipartitions $[10,11]$ for tripartite states and $N$-qubit states [12]. Some other monogamy inequalities are built for multiqubit systems

\footnotetext{
*Corresponding author: mxluo@home.swjtu.edu.cn

Published by the American Physical Society under the terms of the Creative Commons Attribution 4.0 International license. Further distribution of this work must maintain attribution to the author(s) and the published article's title, journal citation, and DOI.
}

in terms of the $\alpha$ th power of entanglement of formation (EOF) $(\alpha \geqslant \sqrt{2})[13-15]$ or the $\alpha$ th power of concurrence $(\alpha \geqslant 2)[16,17]$. Similar results hold for qubit systems with the $\alpha(\alpha \geqslant 2)$ th powers of the Tsallis entropy [18], Rényi entropy [19], and unified entropy [20]. However, most of these well-known entanglement measures [12-15,18,21-24] fail to satisfy the monogamy relation (1) for qubit systems except for the squashed entanglement [25]. A natural problem is to explore which entangled states allow the CKW inequality. One intriguing example is from higher-dimensional quantum systems [18,26,27].

It is difficult to characterize general high-dimensional entangled states because of exponential parameters. One special scenario is from distributive constructions; that is, it allows local tensor structures further regarded as quantum networks [28]. These kinds of multipartite resources are applicable for large-distance quantum communication [29,30], quantum Internet [31,32], quantum secret sharing [26], distributed quantum computing [33], blind quantum computing [34], and quantum sensing [35,36]. This makes intriguing the interesting problem of universal monogamy relations for higher-dimensional systems from quantum networks.

Our motivation in this paper is to explore entangled quantum networks by using quantum entropy toward quantum communication. We firstly prove that the standard CKW monogamy (1) holds for generic entangled quantum networks in terms of EOF and Rényi entropy. This shows features of entanglement distribution for high-dimensional quantum states going beyond qubit entanglement $[10,11,16]$ or single entanglement $[14,22]$. We then apply the proposed entanglement distribution to resolve the maximum-flow minimum-cut (max-flow min-cut) problem for entangled networks even if it fails with single multipartite entanglement [37]. The maxflow min-cut theorem presented here holds for any quantum networks consisting of Einstein-Podolsky-Rosen (EPR) states 
and Greenberger-Horne-Zeilinger (GHZ) states. Moreover, the entanglement entropy implies one way for classifying the configuration for entangled networks under local unitary operations. The present method can be adapted for cyclic networks going beyond recent results [38,39]. These results are interesting in entanglement theory, quantum communication, and quantum information processing.

\section{ENTANGLEMENT MEASURE BASED ON QUANTUM ENTROPY}

In this section, we recall necessary bipartite entanglement measures based on quantum entropies. As an entanglement measure characterizing bipartite entanglement through von Neumann entropy, the entanglement of formation (EOF) $\mathcal{E}$ for a pure state $|\phi\rangle_{\mathrm{AB}}$ on Hilbert space $\mathcal{H}_{\mathrm{A}} \otimes \mathcal{H}_{\mathrm{B}}$ is defined as [40]

$$
\mathcal{E}\left(|\phi\rangle_{\mathrm{AB}}\right)=S\left(\rho_{\mathrm{A}}\right)=-\operatorname{Tr}\left(\rho_{\mathrm{A}} \log _{2} \rho_{\mathrm{A}}\right),
$$

where $\rho_{A}=\operatorname{Tr}_{B}\left(|\phi\rangle_{A B}\langle\phi|\right)$ denotes the density operator of subsystem A obtained by tracing out subsystem B and $S\left(\rho_{\mathrm{A}}\right)$ denotes the von Neumann entropy of system $A$. Note that the von Neumann entropy of two density operators $\rho$ and $\sigma$ satisfies the additivity [41]

$$
S(\rho \otimes \sigma)=S(\rho)+S(\sigma) .
$$

For a bipartite mixed state $\rho_{A B}$ on Hilbert space $\mathcal{H}_{A} \otimes \mathcal{H}_{B}$, EOF is given by

$$
\mathcal{E}\left(\rho_{\mathrm{AB}}\right)=\inf _{\left\{p_{i},\left|\phi_{i}\right\rangle\right\}} \sum_{i} p_{i} \mathcal{E}\left(\left|\phi_{i}\right\rangle_{\mathrm{AB}}\right),
$$

where the infimum takes over all possible pure-state decompositions of $\rho_{\mathrm{AB}}=\sum_{i} p_{i}\left|\phi_{i}\right\rangle_{\mathrm{AB}}\left\langle\phi_{i}\right|$ with $p_{i} \geqslant 0, \sum_{i} p_{i}=1$, and $\left|\phi_{i}\right\rangle_{\mathrm{AB}} \in \mathcal{H}_{\mathrm{A}} \otimes \mathcal{H}_{\mathrm{B}}$.

As a generalization of EOF, the Rényi- $\alpha$ entanglement measure $\mathcal{R}_{\alpha}$ can be defined by using the Rényi- $\alpha$ entropy as [19]

$$
\mathcal{R}_{\alpha}\left(|\phi\rangle_{\mathrm{AB}}\right)=S_{\alpha}\left(\rho_{\mathrm{A}}\right)=\frac{1}{1-\alpha} \log _{2} \operatorname{Tr}\left(\rho_{\mathrm{A}}^{q}\right),
$$

where $S_{\alpha}\left(\rho_{\mathrm{A}}\right)$ denotes the Rényi- $\alpha$ entropy of system A. Similar to the von Neumann entropy, Rényi- $\alpha$ entropy has the additivity [42]

$$
S_{\alpha}(\rho \otimes \sigma)=S_{\alpha}(\rho)+S_{\alpha}(\sigma)
$$

for $\alpha>0$ with $\alpha \neq 1$. For a bipartite mixed state $\rho_{\mathrm{AB}}$, Rényi- $\alpha$ entanglement $\mathcal{R}_{\alpha}$ is defined via the convex-roof extension as [19]

$$
\mathcal{R}_{\alpha}\left(\rho_{\mathrm{AB}}\right)=\inf _{\left\{p_{i},\left|\phi_{i}\right\rangle\right\}} \sum_{i} p_{i} \mathcal{R}_{\alpha}\left(\left|\phi_{i}\right\rangle_{\mathrm{AB}}\right)
$$

with the minimum taking over all possible decompositions of $\rho_{\mathrm{AB}}$.

For a bipartite pure state $|\phi\rangle_{A B}$ on Hilbert space $\mathcal{H}_{A} \otimes$ $\mathcal{H}_{\mathrm{B}}$, The Tsallis- $q$ entanglement measure $\mathcal{T}^{(q)}$ derived from Tsallis- $q$ entropy is defined as [43]

$$
\mathcal{T}^{(q)}\left(|\phi\rangle_{\mathrm{AB}}\right)=S_{q}\left(\rho_{\mathrm{A}}\right)=\frac{1}{q-1}\left(1-\operatorname{Tr}\left(\rho_{\mathrm{A}}^{q}\right)\right),
$$

where $S_{q}\left(\rho_{\mathrm{A}}\right)$ denotes the Tsallis- $q$ entropy of the system A, $q>0$ and $q \neq 1$. $S_{q}(\rho)$ converges to the von Neumann entropy when $q \rightarrow 1$; that is, $\lim _{q \rightarrow 1} S_{q}(\rho)=-\operatorname{Tr} \rho \log _{2}(\rho)=$
$S(\rho)$ for any bipartite operator density $\rho$. For a bipartite mixed state $\rho_{\mathrm{AB}}$, Tsallis- $q$ entropy entanglement $\mathcal{T}^{(q)}$ is defined by

$$
\mathcal{T}^{(q)}\left(\rho_{\mathrm{AB}}\right)=\inf _{\left\{p_{i},\left|\phi_{i}\right\rangle\right\}} \sum_{i} p_{i} \mathcal{T}^{(q)}\left(\left|\phi_{i}\right\rangle_{\mathrm{AB}}\right),
$$

where the infimum is taken over all possible pure-state decompositions of $\rho_{\mathrm{AB}}$.

For $q>1$, the quantum Tsallis entropy satisfies the subadditivity property [44]

$$
S_{q}(\rho \otimes \sigma) \leqslant S_{q}(\rho)+S_{q}(\sigma),
$$

while for $0<q<1$, it satisfies

$$
S_{q}(\rho \otimes \sigma) \geqslant S_{q}(\rho)+S_{q}(\sigma) .
$$

Different from all the stated entropies, there exists a generalized entropy, i.e., unified $(q, s)$ entropy [45], which involves two real parameters $q$ and $s$ :

$$
S_{q, s}(\rho)=\frac{1}{(1-q) s}\left(\operatorname{Tr}\left(\rho^{q}\right)^{s}-1\right),
$$

where $q, s \geqslant 0$ and $q \neq 1, s \neq 0$. This entropy includes Rényi- $\alpha$ entropy [46] and Tsallis- $q$ entropy [47] as special cases of $s \rightarrow 0$ and $s \rightarrow 1$, respectively. Moreover, for $q \rightarrow 1$, it converges to the von Neumann entropy [41]. Using the unified $(q, s)$ entropy, the unified $(q, s)$ entanglement measure is defined for a bipartite pure state $|\phi\rangle_{A B}$ on Hilbert space $\mathcal{H}_{\mathrm{A}} \otimes \mathcal{H}_{\mathrm{B}}$ by [20]

$$
\mathcal{U}^{(q, s)}\left(|\phi\rangle_{\mathrm{AB}}\right)=S_{q, s}\left(\rho_{\mathrm{A}}\right)
$$

for each $q, s \geqslant 0$. For a bipartite mixed state $\rho_{\mathrm{AB}}$, its entanglement measure is given by

$$
\mathcal{U}^{(q, s)}\left(\rho_{\mathrm{AB}}\right)=\inf _{\left.\left\{p_{i}, \mid \phi_{i}\right)\right\}} \sum_{i} p_{i} \mathcal{U}^{(q, s)}\left(\left|\phi_{i}\right\rangle_{\mathrm{AB}}\right),
$$

where the infimum is taken over all possible pure-state decompositions of $\rho_{\mathrm{AB}}$.

For $q \geqslant 1$ and $s \geqslant 0$, the quantum unified $(q, s)$ entropy satisfies the subadditivity property [48]

$$
S_{q, s}(\rho \otimes \sigma) \leqslant S_{q, s}(\rho)+S_{q, s}(\sigma),
$$

while for $0<q<1$ and $s>0$ it satisfies

$$
S_{q, s}(\rho \otimes \sigma) \geqslant S_{q, s}(\rho)+S_{q, s}(\sigma) .
$$

From all the aforementioned bipartite entanglement measures, they are subadditive; that is,

$$
\mathcal{Q}(\rho \otimes \sigma) \leqslant \mathcal{Q}(\rho)+\mathcal{Q}(\sigma),
$$

where $\mathcal{Q}$ can be any one of $\mathcal{E}, \mathcal{R}_{\alpha}, \mathcal{T}^{(q)}$, and $\mathcal{U}^{(q, s)}$.

\section{ENTANGLEMENT MONOGAMY RELATION OF QUANTUM NETWORKS}

In comparison to single entanglement, a tripartite quantum network consists of various bipartite entangled (BE) states and GHZ states, as shown in Fig. 1. Here, the local unitary operations may entangle these independent states into an entanglement in the global version. This generates high-dimensional quantum entangled states that are important 


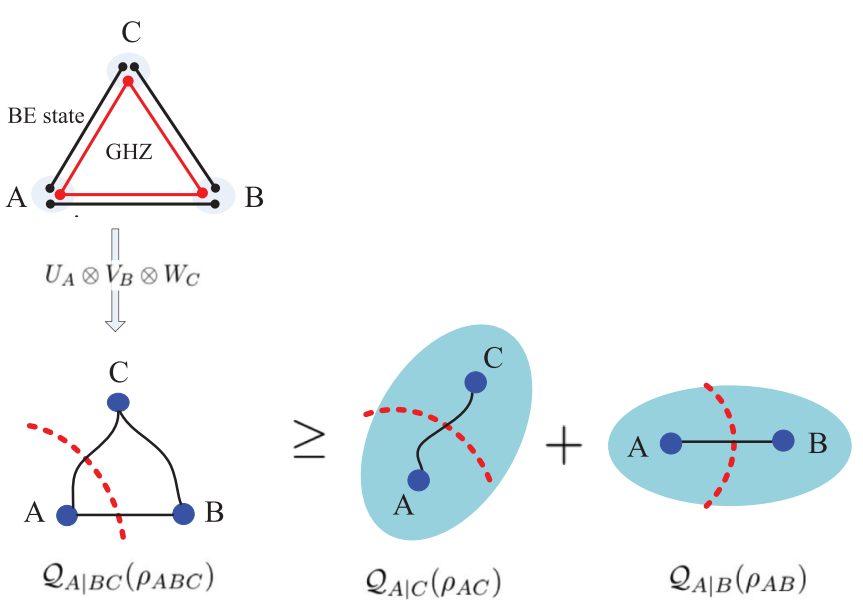

FIG. 1. A monogamy relation of tripartite entanglement inspired by a tripartite quantum network consisting of one GHZ state and three arbitrary bipartite entangled (BE) states. $U, V$, and $W$ are local unitary operations. $\mathcal{Q}$ is entanglement measure defined in the inequality (1).

resources in quantum information processing. Characterizing the general high-dimensional quantum systems is difficult because of the exponential number of parameters. Our motivation here is to feature quantum networks via entanglement distribution by using quantum entropy. The high-dimensional entanglement inspired by quantum networks allows local tensor decomposition that provides the possibility for evaluating new monogamy relations based on von Neumann entropy [41] and Rényi entropy [46], Tsallis entropy [47], and unified entropy [45].

\section{A. Quantum network with generalized GHZ states and EPR states}

Consider an $n$-partite entangled quantum network $\mathcal{N}_{q}(\mathcal{A}, \xi)$, where $\mathcal{A}$ denotes parties $\mathrm{A}_{1}, \ldots, \mathrm{A}_{n}$, and $\xi$ denotes entangled states. Assume any two parties $\mathrm{A}_{i}$ and $\mathrm{A}_{j}$ share the states $\varrho_{i j}\left(\theta_{1}\right), \ldots, \varrho_{i j}\left(\theta_{s}\right), \sigma_{i j}\left(\varphi_{1}\right), \ldots, \sigma_{i j}\left(\varphi_{t}\right)$, and $\delta_{i j}\left(\vartheta_{1}\right), \ldots, \delta_{i j}\left(\vartheta_{k}\right)$, where $\varrho(\theta)$ denotes the density matrix of generalized EPR state [49] $|\phi(\theta)\rangle=\cos \theta|00\rangle+\sin \theta|11\rangle$, $\sigma(\varphi)$ is the density matrix of generalized GHZ state [50] $|\phi(\varphi)\rangle=\cos \varphi|0\rangle^{\otimes m}+\sin \varphi|1\rangle^{\otimes m}$ with any integer $m \geqslant 3$, and $\left.\delta(\vartheta)=\cos ^{2} \vartheta|00\rangle\left\langle 00\left|+\sin ^{2} \vartheta\right| 11\right\rangle\langle 11|\right)$ is the reduced density matrix of any two subsystems obtained by tracing out other subsystems in a generalized GHZ state. Here, each party can perform any local unitary operations to entangle the local systems. With this formation, the total system may be regarded as an $n$-partite entanglement in high-dimensional Hilbert spaces. Informally, we show that the entanglement distribution for quantum networks with local unitary operations satisfies the monogamy inequality (1).

Theorem 1. The entanglement distribution of quantum network $\mathcal{N}_{q}(\mathcal{A}, \xi)$ is given by

$$
\mathcal{Q}_{\mathrm{A}_{i} \mid \overline{\mathrm{A}_{i}}}\left(\rho_{\mathrm{A}_{1} \ldots \mathrm{A}_{n}}\right) \geqslant \sum_{j=1, j \neq i}^{N} \mathcal{Q}_{\mathrm{A}_{i} \mid \mathrm{A}_{j}}\left(\rho_{\mathrm{A}_{i} \mathrm{~A}_{j}}\right),
$$

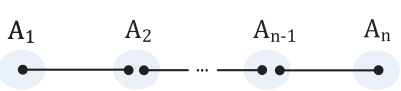

(a)

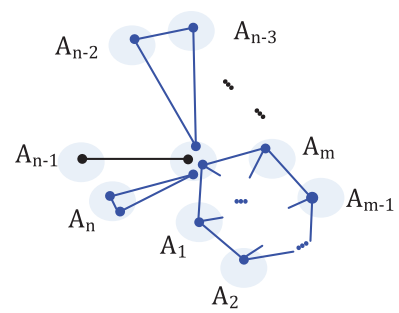

(c)

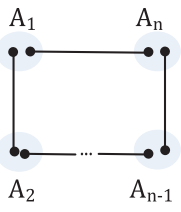

(b)

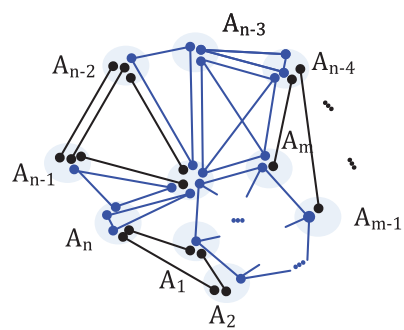

(d)
FIG. 2. Schematic quantum network consisting of generalized EPR states or generalized GHZ states. (a) A chain quantum network consisting of $n-1$ generalized EPR states. (b) A cyclic quantum network consisting of $n$ generalized EPR states. (c) A star quantum network consisting of generalized EPR states and generalized GHZ states. (d) A general quantum network consisting of generalized EPR states and generalized GHZ states.

where $\mathcal{Q}$ denotes the entanglement measure of EOF or Rényi$\alpha$ entropy, $\mathcal{Q}_{\mathrm{X} \mid \mathrm{Y}}$ is an entanglement measure of a composite quantum system with respect to the bipartite cut between $X$ and $\mathrm{Y}$, and $\overline{\mathrm{A}_{i}}$ denotes all parties except for $\mathrm{A}_{i}$. The equality holds if and only if all GHZ states are being shared by two parties.

Proof. As shown in Fig. 2, we illustrate quantum networks consisting of generalized EPR states and GHZ states; these include a chain network [Fig. 2(a)], cyclic network [Fig. 2(b)], star network [Fig. 2(c)], and general network [Fig. 2(d)]. Now, we prove the monogamy for general network $\mathcal{N}_{q}(\mathcal{A}, \xi)$ as shown in Fig. 2(d). Suppose that any two parties $A_{i}$ and $A_{j}$ share the quantum state $\xi_{i j}$ given by

$$
\xi_{i j}=\bigotimes_{s=1}^{s_{j}} \varrho_{i j}\left(\theta_{s}\right) \bigotimes_{t=1}^{t_{j}} \sigma_{i j}\left(\varphi_{t}\right) \bigotimes_{k=1}^{k_{j}} \delta_{i j}\left(\vartheta_{k}\right),
$$

where $\varrho\left(\theta_{s}\right)$ is the reduced density matrix of generalized EPR state [49] $\left|\phi\left(\theta_{s}\right)\right\rangle=\cos \theta_{s}|00\rangle+\sin \theta_{s}|11\rangle$, $\sigma\left(\varphi_{t}\right)$ is the reduced density matrix of generalized GHZ state [50] $\left|\phi\left(\varphi_{t}\right)\right\rangle=\cos \varphi_{t}|0\rangle^{\otimes m}+\sin \varphi_{t}|1\rangle^{\otimes m}$, and $\delta\left(\vartheta_{k}\right)=$ $\left.\cos ^{2} \vartheta_{k}|00\rangle\left\langle 00\left|+\sin ^{2} \vartheta_{k}\right| 11\right\rangle\langle 11|\right)$ is the reduced density matrix of any two subsystems by tracing out the remaining subsystems in a multipartite generalized GHZ state. Herein, $s_{j}, t_{j}$, and $k_{j}$ denote the numbers of $\varrho\left(\theta_{s}\right), \sigma\left(\varphi_{t}\right)$, and $\delta\left(\vartheta_{k}\right)$ that are shared by any two parties $A_{i}$ and $A_{j}$, respectively. Then, the total state $\rho_{\mathrm{A}_{1} \ldots \mathrm{A}_{n}}$ of an $n$-partite system is given by

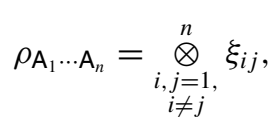

where $\xi_{i j}$ has the form of Eq. (19). 
Assume that any party $\mathrm{A}_{i}$ is entangled with $N$ parties of $A_{j}$ 's. From Eq. (2), we get

$$
\begin{aligned}
& \mathcal{E}_{\mathrm{A}_{i} \mid \overline{\mathrm{A}_{i}}}\left(\rho_{\mathrm{A}_{1} \cdots \mathrm{A}_{n}}\right)=S\left(\rho_{\mathrm{A}_{i}}\right) \\
& \left.=S\left(\bigotimes_{j=1}^{N}\left(\bigotimes_{s=1}^{s_{j}} \rho_{i ; j}\left(\theta_{s}\right){\stackrel{\bigotimes}{t_{j}}}_{t=1} \rho_{i ; j}\left(\varphi_{t}\right){\stackrel{\bigotimes}{k_{j}}}_{k=1}^{k_{i ; j}} \rho_{k}\right)\right)\right) \\
& =\sum_{j=1}^{N}\left(\sum_{s=1}^{s_{j}} S\left(\rho_{i ; j}\left(\theta_{s}\right)\right)+\sum_{t=1}^{t_{j}} S\left(\rho_{i ; j}\left(\varphi_{t}\right)\right)\right) \\
& +\sum_{j=1}^{N} \sum_{k=1}^{k_{j}} S\left(\rho_{i ; j}\left(\vartheta_{k}\right)\right),
\end{aligned}
$$

where Eq. (21) follows from the additivity of von Neumann entropy in Eq. (3). Here, $\rho_{i ; j}\left(\theta_{s}\right)$ is the reduced density matrix of the subsystems owned by $\mathrm{A}_{i}$, which is obtained by tracing out all the subsystems owned by $\mathbf{A}_{j}$ in a generalized EPR state $\left|\phi\left(\theta_{s}\right)\right\rangle_{i, j} . \rho_{i ; j}\left(\varphi_{t}\right)$ is the reduced density operator of the subsystem owned by $A_{i}$ with respect to the generalized GHZ state shared by two parties $A_{i}$ and $A_{j} . \rho_{i ; j}\left(\vartheta_{k}\right)$ is the reduced density matrix of the subsystems owned by $\mathrm{A}_{i}$, which is obtained by tracing out the other subsystems in a generalized GHZ state shared by multipartite parties.

Note that the entanglement shared by the parties $A_{i}$ and $A_{j}$ is generated by generalized EPR states and GHZ states. It is sufficient to prove that

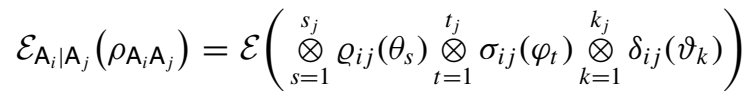

$$
\begin{aligned}
& \leqslant \mathcal{E}\left(\varrho_{i j}\left(\theta_{s}\right)\right)+\mathcal{E}\left({\stackrel{\otimes}{t_{j}}}_{t=1}^{t_{i j}} \sigma_{i j}\left(\varphi_{t}\right)\right) \\
& =\sum_{s=1}^{s_{j}} S\left(\rho_{i ; j}\left(\theta_{s}\right)\right)+\sum_{t=1}^{t_{j}} S\left(\rho_{i ; j}\left(\varphi_{t}\right)\right) .
\end{aligned}
$$

From the additivity of the entanglement measure $\mathcal{E}$ in Eq. (17) and $\mathcal{E}\left(\delta_{i j}\left(\vartheta_{k}\right)\right)=0$, the inequality (22) follows. Equation (23) is derived from the definition of the entanglement measure $\mathcal{E}$ in Eq. (2). Combining Eq. (21) with Eq. (23) yields

$$
\begin{aligned}
\mathcal{E}_{\mathrm{A}_{i} \mid \overline{\mathrm{A}_{i}}}\left(\rho_{\mathrm{A}_{1} \cdots \mathrm{A}_{n}}\right) & \geqslant \sum_{j=1, j \neq i}^{N} \mathcal{E}_{\mathrm{A}_{i} \mid \mathrm{A}_{j}}\left(\rho_{\mathrm{A}_{i} \mathrm{~A}_{j}}\right) \\
& =\sum_{j=1, j \neq i}^{n} \mathcal{E}_{\mathrm{A}_{i} \mid \mathrm{A}_{j}}\left(\rho_{\mathrm{A}_{i} \mathrm{~A}_{j}}\right) .
\end{aligned}
$$

Note that for $k_{j}=0$, i.e., all $m$-qubit GHZ states are shared by two parties, we have

$$
\mathcal{E}_{\mathrm{A}_{i} \mid \overline{\mathrm{A}_{i}}}\left(\rho_{\mathrm{A}_{1} \ldots \mathrm{A}_{n}}\right)=\sum_{j=1}^{N}\left(\sum_{s=1}^{s_{j}} S\left(\rho_{i ; j}\left(\theta_{s}\right)\right)+\sum_{t=1}^{t_{j}} S\left(\rho_{i ; j}\left(\varphi_{t}\right)\right)\right) .
$$

Moreover, we obtain

$$
\mathcal{E}_{\mathrm{A}_{i} \mid \mathrm{A}_{j}}\left(\rho_{\mathrm{A}_{i} \mathrm{~A}_{j}}\right)=\sum_{j=1}^{N}\left(\sum_{s=1}^{s_{j}} S\left(\rho_{i ; j}\left(\theta_{s}\right)\right)+\sum_{t=1}^{t_{j}} S\left(\rho_{i ; j}\left(\varphi_{t}\right)\right)\right) .
$$

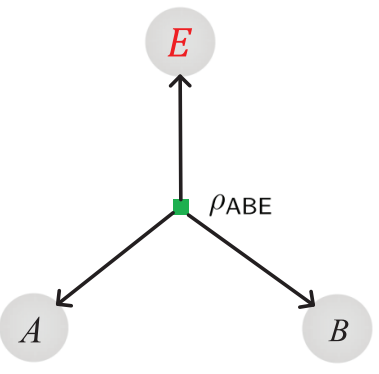

(a)

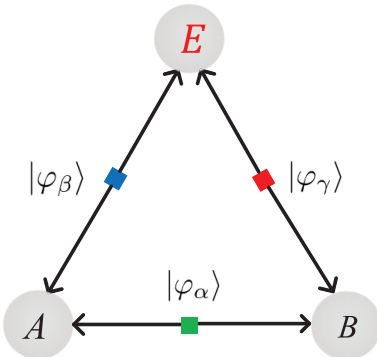

(b)
FIG. 3. Device-independent quantum key distribution with an attacker E. (a) The standard attack model. (b) The attack model based on the triangle network.

So, combining Eqs. (26) and (27), we get

$$
\mathcal{E}_{\mathrm{A}_{i} \mid \overline{\mathrm{A}_{i}}}\left(\rho_{\mathrm{A}_{1} \cdots \mathrm{A}_{n}}\right)=\sum_{j=1, j \neq i}^{n} \mathcal{E}_{\mathrm{A}_{i} \mid \mathrm{A}_{j}}\left(\rho_{\mathrm{A}_{i} \mathrm{~A}_{j}}\right)
$$

Similarly, the inequality (18) holds for the Rényi- $\alpha$ entropy entanglement in Eq. (5) from the additivity of Rényi- $\alpha$ entropy in Eq. (6), i.e.,

$$
\mathcal{R}_{\alpha}^{\mathrm{A}_{i} \mid \overline{\mathrm{A}_{i}}}\left(\rho_{\mathrm{A}_{1} \ldots \mathrm{A}_{n}}\right) \geqslant \sum_{j=1, j \neq i}^{N} \mathcal{R}_{\alpha}^{\mathrm{A}_{i} \mid \mathrm{A}_{j}}\left(\rho_{\mathrm{A}_{i} \mathrm{~A}_{j}}\right) .
$$

This has completed the proof.

Theorem 1 indicates a mutually exclusive relation of quantum networks between any pair of parties $A_{i}$ and $A_{j}$, which goes beyond the single entanglement of GHZ states being ruled out by the monogamy inequality (1). The more interesting part is that the present result provides a generic monogamous relation for high-dimensional entanglement beyond qubit states [10-12,16,18-20]. It is also stronger than the existence result of high-dimensional entanglement [26]. Theorem 1 may provide a better-than-single-entanglement performance in quantum tasks. One example is device-independent quantum key distribution [5] where the eavesdropper's attacks are basically limited by the monogamy of entanglement.

Example 1. Consider a device-independent quantum key distribution model with entanglement $\rho_{\mathrm{AB}}$ [6]. There is an eavesdropper Eve, represented by $\mathrm{E}$, who may be correlated with $\rho_{\mathrm{AB}}$. The total state is then denoted by $\rho_{\mathrm{ABE}}$ satisfying $\operatorname{Tr}_{\mathrm{E}}\left(\rho_{\mathrm{ABE}}\right)=\rho_{\mathrm{AB}}$, as shown in Fig. 3(a). Eve is assumed to control the source or measurement devices in a relaxed model. The entanglement between Eve and Alice satisfies the monogamy relation [16]

$$
\mathcal{E}_{\mathrm{A} \mid \mathrm{E}}\left(\rho_{\mathrm{AE}}\right) \leqslant\left(\mathcal{E}_{\mathrm{A} \mid \mathrm{BC}}^{\sqrt{2}}\left(\rho_{\mathrm{ABE}}\right)-\mathcal{E}_{\mathrm{A} \mid \mathrm{B}}^{\sqrt{2}}\left(\rho_{\mathrm{AB}}\right)\right)^{\frac{1}{\sqrt{2}}},
$$

where $\mathcal{E}$ represents the EOF measure. The inequality (30) provides a monogamy relation for the information leakage in a device-independent model.

Instead, the eavesdropper may hold local systems correlated with $\rho_{\mathrm{AB}}$. That is, Eve fakes entangled sources $\left|\varphi_{\beta}\right\rangle$ and $\left|\varphi_{\gamma}\right\rangle$ between her device and Alice's and Bob's respective devices, as shown in Fig. 3(b). This generates a triangle quantum 


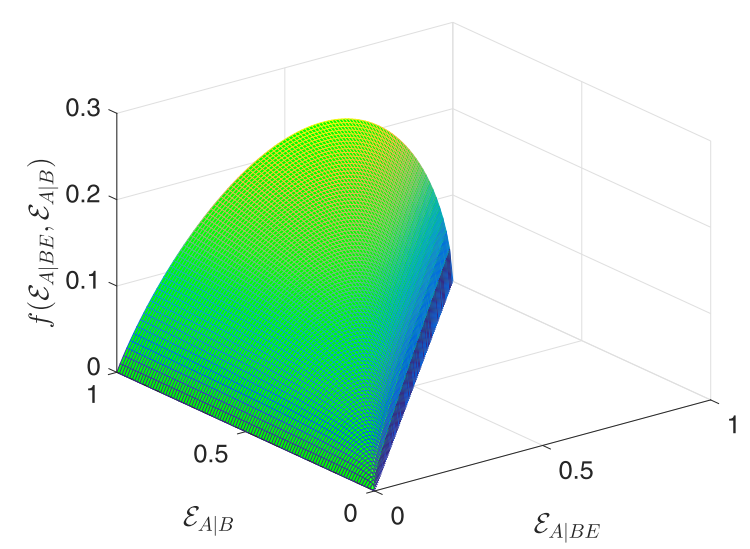

FIG. 4. Schematic difference between the upper bounds (30) and (31).

network which allows from Theorem 1 the relation

$$
\mathcal{E}_{\mathrm{A} \mid \mathrm{E}}\left(\rho_{\mathrm{AE}}\right) \leqslant \mathcal{E}_{\mathrm{A} \mid \mathrm{BE}}\left(\rho_{\mathrm{ABE}}\right)-\mathcal{E}_{\mathrm{A} \mid \mathrm{B}}\left(\rho_{\mathrm{AB}}\right) .
$$

The difference between the upper bounds (30) and (31) is denoted as

$$
f\left(\mathcal{E}_{\mathrm{A} \mid \mathrm{BE}}, \mathcal{E}_{\mathrm{A} \mid \mathrm{B}}\right)=\left(\mathcal{E}_{\mathrm{A} \mid \mathrm{BE}}{ }^{\sqrt{2}}-\mathcal{E}_{\mathrm{A} \mid \mathrm{B}}^{\sqrt{2}}\right)^{\frac{1}{\sqrt{2}}}-\left(\mathcal{E}_{\mathrm{A} \mid \mathrm{BE}}-\mathcal{E}_{\mathrm{A} \mid \mathrm{B}}\right),
$$

where $\mathcal{E}_{\mathrm{A} \mid \mathrm{BE}}$ and $\mathcal{E}_{\mathrm{A} \mid \mathrm{B}}$ satisfy the inequality $\mathcal{E}_{\mathrm{A} \mid \mathrm{BE}}>\mathcal{E}_{\mathrm{A} \mid \mathrm{B}}$. A schematic demonstration is given to state the difference between the upper bounds (30) and (31) as shown in Fig. 4. Obviously, $f\left(\mathcal{E}_{\mathrm{A} \mid \mathrm{BE}}, \mathcal{E}_{\mathrm{A} \mid \mathrm{B}}\right)$ is always positive; this indicates that the inequality (31) provides a stronger constraint for the information leakage in a device-independent model, which makes it harder for the attacker to carry out the attack.

\section{B. General quantum networks}

In this section, we extend Theorem 1 to general quantum networks.

\section{Quantum network with W states}

Consider a genuine tripartite entangled $\mathrm{W}$ state [51]:

$$
|W\rangle=\frac{1}{\sqrt{3}}(|100\rangle+|010\rangle+|001\rangle) .
$$

It is easy to obtain

$$
\begin{aligned}
\mathcal{E}\left(|W\rangle_{\mathrm{A} \mid \mathrm{B}_{1} \mathrm{~B}_{2}}\right) & =H\left(\frac{1}{3}\right) \approx 0.918, \\
\mathcal{E}\left(\rho_{\mathrm{AB}_{i}}\right) & =H\left(\frac{1+\sqrt{1-C^{2}\left(\rho_{\mathrm{AB}_{i}}\right)}}{2}\right) \approx 0.55,
\end{aligned}
$$

where $\mathcal{E}$ represents EOF in Eq. (2), $H(x)$ is the binary Shannon entropy given $H(x)=-x \log _{2} x-(1-x) \log _{2}(1-$ $x)$ for $0 \leqslant x \leqslant 1$ [41], and $C\left(\rho_{\mathrm{AB}_{i}}\right)=\frac{2}{3}(i=1,2)$ is the concurrence [52] of the two-qubit mixed state $\rho_{\mathrm{AB}_{i}}$. From Eq. (34), we have

$$
\mathcal{E}\left(|W\rangle_{{\mathrm{A} \mid \mathrm{B}_{1} \mathrm{~B}_{2}}_{2}} \leqslant \mathcal{E}\left(\rho_{\mathrm{AB}_{1}}\right)+\mathcal{E}\left(\rho_{\mathrm{AB}_{2}}\right) ;\right.
$$

that is, EOF $\mathcal{E}$ of the $\mathrm{W}$ state is not monogamous. However, we prove that the monogamy relation of $\mathcal{E}$ holds for quantum networks consisting of GHZ states and $\mathrm{W}$ states. The monogamy of the quantum network reaches a good saturation in the case that the quantum network consists of $|W\rangle^{\otimes 5} \otimes|G H Z\rangle$, i.e., five pairs of the W state and one GHZ state are shared by three parties, simultaneously. The main reason is as follows. For the GHZ state, we have

$$
\begin{aligned}
\mathcal{E}\left(|\mathrm{GHZ}\rangle_{\mathrm{A} \mid \mathrm{BC}}\right) & =1, \\
\mathcal{E}\left(\rho_{\mathrm{AB}}\right) & =\mathcal{E}\left(\rho_{\mathrm{AC}}\right)=0 .
\end{aligned}
$$

From Eqs. (34) and (36), we get the inequality

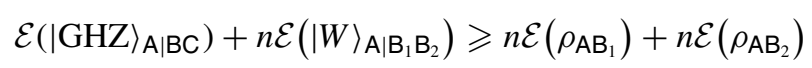

for $n \leqslant 5$. The inequality (37) is optimal in the case of $n=5$, i.e.,

$$
1+5 H\left(\frac{1}{3}\right) \geqslant 5 \mathcal{E}\left(\rho_{\mathrm{AB}_{1}}\right)+5 \mathcal{E}\left(\rho_{\mathrm{AB}_{2}}\right) .
$$

From the inequality (38), we extend Theorem 1 to the network consisting of EPR states, GHZ states, and W states. Consider an $n$-partite entangled quantum network $\mathcal{N}_{q_{1}}(\mathcal{A}, \xi)$, where $\mathcal{A}$ denotes parties $A_{1}, \ldots, A_{n}$, and $\xi$ denotes entangled states. Assume any two parties $\mathrm{A}_{i}$ and $\mathrm{A}_{j}$ share the state $\xi_{i j}$ given by

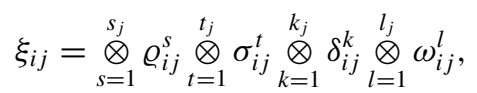

where $\varrho$ denotes the density matrix of EPR state $[49]|\Phi\rangle=$ $\frac{1}{\sqrt{2}}(|00\rangle+|11\rangle) . \sigma$ is the density matrix of GHZ state [50] $|\Psi\rangle=\frac{1}{\sqrt{2}}(|000\rangle+|111\rangle) \cdot \delta=\frac{1}{\sqrt{2}}(|00\rangle\langle 00|+| 11\rangle\langle 11|)$ is the reduced density matrix of any two subsystems in a GHZ state, and $\omega=\frac{2}{3}|00\rangle\left\langle 00\left|+\frac{1}{3}\right| 11\right\rangle\langle 11|$ is the reduced density matrix of any two subsystems in the $\mathrm{W}$ state in Eq. (33). $s_{j}, t_{j}, k_{j}$, and $l_{j}$ denote the numbers of $\varrho, \sigma, \delta$, and $\omega$, respectively. All these entangled states are shared by any two parties $A_{i}$ and $\mathrm{A}_{j}$. Moreover, $l_{j}$ and $k_{j}$ satisfy $l_{j} \leqslant 5 k_{j}$. Thus the total state $\rho_{\mathrm{A}_{1} \cdots \mathrm{A}_{n}}$ of an $n$-partite system is given by

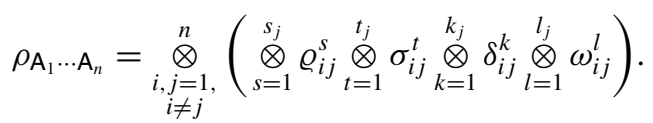

Theorem 2. The entanglement distribution of quantum network $\mathcal{N}_{q_{1}}$ satisfies

$$
\mathcal{E}_{\mathrm{A}_{i} \mid \overline{A_{i}}}\left(\rho_{\mathrm{A}_{1} \cdots \mathrm{A}_{n}}\right) \geqslant \sum_{j=1, j \neq i}^{n} \mathcal{E}_{\mathrm{A}_{i} \mid \mathrm{A}_{j}}\left(\rho_{\mathrm{A}_{i} \mathrm{~A}_{j}}\right),
$$

where $\mathcal{E}_{\mathrm{X} \mid \mathrm{Y}}$ represents $\mathrm{EOF}$ of a bipartite system $\mathrm{X}$ and $\mathrm{Y}$, and $\overline{\mathrm{A}_{i}}$ denotes all parties except for $\mathrm{A}_{i}$.

Proof. Assume that any party $\mathrm{A}_{i}$ is entangled with $N$ parties of $\mathrm{A}_{j}$ 's. The total numbers of EPR, GHZ, and W states are $n_{1}, n_{2}$, and $n_{3}$, respectively. Any two parties $\mathrm{A}_{i}$ and $\mathrm{A}_{j}$ share $s_{j}$ pairs of EPR states $\varrho, t_{j}$ pairs of GHZ states $\sigma, k_{j}$ pairs of $\delta$, and $l_{j}$ pairs of $\omega$. As a result, it is easy to obtain that $\sum_{j=1}^{N} s_{j}=n_{1}, \sum_{j=1}^{N} t_{j}=n_{2}^{\prime}, \sum_{k=1}^{N} k_{j}=2\left(n_{2}-n_{2}^{\prime}\right)$, and $\sum_{l=1}^{N} l_{j}=2 n_{3}$, where $n_{2}^{\prime}$ is the number of GHZ states shared by two parties. There are $n_{2}-n_{2}^{\prime}$ pairs of GHZ states shared by three parties. From the inequality (38), the entanglement 
distribution holds for a quantum network consisting of GHZ states and $\mathrm{W}$ states from $l_{j} \leqslant 5 k_{j}$. This yields the inequality $n_{3} \leqslant 5\left(n_{2}-n_{2}^{\prime}\right)$. From Eq. (2), we get

$$
\begin{aligned}
\mathcal{E}_{\mathrm{A}_{i} \mid \overline{\mathrm{A}_{i}}}\left(\rho_{\mathrm{A}_{1} \cdots \mathrm{A}_{n}}\right) & =S\left(\rho_{\mathrm{A}_{i}}\right) \\
& =S\left(\left(\frac{\mathbb{1}}{2}\right)^{\otimes n_{1}} \otimes\left(\frac{\mathbb{1}}{2}\right)^{\otimes n_{2}} \otimes \omega^{\otimes n_{3}}\right) \\
& =n_{1} S\left(\frac{\mathbb{1}}{2}\right)+n_{2} S\left(\frac{\mathbb{1}}{2}\right)+n_{3} S(\omega) \\
& =n_{1}+n_{2}+n_{3} H\left(\frac{1}{3}\right),
\end{aligned}
$$

where Eq. (42) follows from the additivity of the von Neumann entropy in Eq. (3). In the equality (43), we have used $S\left(\frac{\mathbb{1}}{2}\right)=1$ and $S(\omega)=H\left(\frac{1}{3}\right)$.

Moreover, we have

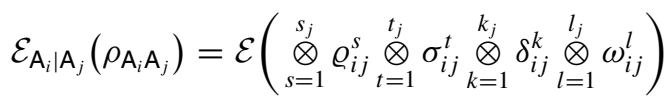

$$
\begin{aligned}
& \leqslant \mathcal{E}\left(\stackrel{\bigotimes}{s=1}_{s_{j}} \varrho_{i j}^{s}\right)+\mathcal{E}\left(\stackrel{\bigotimes}{t=1}_{t}^{t_{j}} \sigma_{i j}^{t}\right)+\mathcal{E}\left(\bigotimes_{l=1}^{l_{j}} \omega_{i j}^{l}\right) \\
& \leqslant \sum_{s=1}^{s_{j}} S\left(\frac{\mathbb{1}}{2}\right)+\sum_{t=1}^{t_{j}} S\left(\frac{\mathbb{1}}{2}\right)+\sum_{l=1}^{l_{j}} \mathcal{E}\left(\omega_{i j}^{l}\right) \\
& =\sum_{s=1}^{s_{j}} 1+\sum_{t=1}^{t_{j}} 1+\sum_{l=1}^{l_{j}} \mathcal{E}(\omega) \\
& =s_{j}+t_{j}+l_{j} \mathcal{E}(\omega) \text {, }
\end{aligned}
$$

where the inequality (44) follows from the additivity of EOF in Eq. (17). The inequality (45) is from the definition of EOF in Eq. (2) and the additivity of EOF in Eq. (17). We therefore have

$$
\begin{aligned}
\sum_{j=1}^{N} \mathcal{E}_{\mathrm{A}_{i} \mid \mathrm{A}_{j}}\left(\rho_{\mathrm{A}_{i} \mathrm{~A}_{j}}\right) & \leqslant \sum_{j=1}^{N}\left(s_{j}+t_{j}+l_{j} \mathcal{E}(\omega)\right) \\
& =n_{1}+n_{2}^{\prime}+2 n_{3} \mathcal{E}(\omega) \\
& \leqslant n_{1}+n_{2}^{\prime}+\frac{n_{3}}{5}+n_{3} H\left(\frac{1}{3}\right) \\
& \leqslant n_{1}+n_{2}+n_{3} H\left(\frac{1}{3}\right),
\end{aligned}
$$

where the equality (47) is derived from the equalities $\sum_{j=1}^{N} s_{j}=n_{1}, \sum_{j=1}^{N} t_{j}=n_{2}^{\prime}$, and $\sum_{j=1}^{N} l_{j}=2 n_{3}$. The inequality (48) follows from the inequality $5 \times 2 \mathcal{E}(\omega) \leqslant 1+$ $5 H\left(\frac{1}{3}\right)$, which is from the inequality (38). The inequality (49) holds for the inequality $n_{3} \leqslant 5\left(n_{2}-n_{2}^{\prime}\right)$. Finally, we obtain

$$
\begin{aligned}
\mathcal{E}_{\mathrm{A}_{i} \mid \overline{\mathrm{A}_{i}}}\left(\rho_{\mathrm{A}_{1} \ldots \mathrm{A}_{n}}\right) & \geqslant \sum_{j=1, j \neq i}^{N} \mathcal{E}_{\mathrm{A}_{i} \mid \mathrm{A}_{j}}\left(\rho_{\mathrm{A}_{i} \mathrm{~A}_{j}}\right) \\
& =\sum_{j=1, j \neq i}^{n} \mathcal{E}_{\mathrm{A}_{i} \mid \mathrm{A}_{j}}\left(\rho_{\mathrm{A}_{i} \mathrm{~A}_{j}}\right) .
\end{aligned}
$$

This completes the proof of Theorem 2 .

\section{Quantum network with arbitrary bipartite entangled pure states}

Consider an $n$-partite entangled quantum network $\mathcal{N}_{q_{2}}(\mathcal{A}, \xi)$, where any two parties $\mathrm{A}_{i}$ and $\mathrm{A}_{j}$ share $s_{j}$ pairs of arbitrary bipartite entangled pure states $\left|\varphi^{1}\right\rangle_{i j},\left|\varphi^{2}\right\rangle_{i j}, \ldots,\left|\varphi^{s}\right\rangle_{i j}$. The joint state shared by two parties $\mathrm{A}_{i}$ and $\mathrm{A}_{j}$ is denoted by $\otimes_{s=1}^{s_{j}}\left|\varphi^{s}\right\rangle_{i j}$. The total state $\rho_{\mathrm{A}_{1} \ldots \mathrm{A}_{n}}$ of $\mathcal{N}_{q_{2}}(\mathcal{A}, \xi)$ is given by

$$
\rho_{\mathrm{A}_{1} \cdots \mathrm{A}_{n}}=\bigotimes_{\substack{i, j=1, j=1 \\ i \neq j}}^{n} \bigotimes_{s=1}^{s_{j}}\left|\varphi^{s}\right\rangle_{i j} .
$$

We prove a strong monogamy relation for quantum network $\mathcal{N}_{q_{2}}$.

Theorem 3. The entanglement distribution of quantum network $\mathcal{N}_{q_{2}}$ satisfies

$$
\mathcal{Q}_{\mathrm{A}_{i} \mid \overline{\mathrm{A}_{i}}}\left(\rho_{\mathrm{A}_{1} \ldots \mathrm{A}_{n}}\right)=\sum_{j=1, j \neq i}^{n} \mathcal{Q}_{\mathrm{A}_{i} \mid \mathrm{A}_{j}}\left(\rho_{\mathrm{A}_{i} \mathrm{~A}_{j}}\right),
$$

where $\mathcal{Q}_{\mathrm{A}_{i} \mid \overline{\mathrm{A}_{i}}}$ and $\mathcal{Q}_{\mathrm{A}_{i} \mid \mathrm{A}_{j}}$ are defined in Theorem 1 .

Proof. Assume that there exist $N$ parties $\mathrm{A}_{j}$ who are entangled with the party $A_{i}$. Note that any two parties $A_{i}$ and $A_{j}$ share the state of $\otimes_{s=1}^{s_{j}}\left|\varphi^{s}\right\rangle_{i j}$. It is sufficient to prove that

$$
\begin{aligned}
\mathcal{E}_{\mathrm{A}_{i} \mid \overline{\mathrm{A}_{i}}}\left(\rho_{\mathrm{A}_{1} \cdots \mathrm{A}_{n}}\right) & =\mathcal{E}\left(\bigotimes_{j=1}^{N} \bigotimes_{s=1}^{s_{j}}\left|\varphi^{s}\right\rangle_{i j}\right) \\
& =S\left(\rho_{i}^{j}\right) \\
& =S\left(\bigotimes_{j=1}^{N} \bigotimes_{s=1}^{s_{j}} \rho_{i ; j}^{s}\right) \\
= & \sum_{j=1}^{N} \sum_{s=1}^{s_{j}} S\left(\rho_{i ; j}^{s}\right),
\end{aligned}
$$

where the equality (53) is due to the systems $\rho_{i ; j}^{s}$ being uncorrelated, i.e., $\rho_{i}^{j}=\otimes_{j=1}^{N} \otimes_{s=1}^{s_{j}} \rho_{i ; j}^{s}$, and $\rho_{i ; j}^{s}$ is the reduced density matrix of the subsystems owned by the party $A_{i}$ by tracing out the subsystems owned by $A_{j}$ in arbitrary bipartite entangled pure state $\left|\varphi^{s}\right\rangle_{i j}\left\langle\varphi^{s}\right|$. The equality (54) is obtained by iteratively using the additivity of von Neumann entropy in Eq. (3).

Moreover, the entanglement shared by two parties $A_{i}$ and $\mathrm{A}_{j}$ is from entangled sources of $\otimes_{s=1}^{s_{j}}\left|\varphi^{s}\right\rangle_{i j}$. According to Eq. (2), we obtain

$$
\begin{aligned}
\sum_{j=1}^{N} \mathcal{E}_{\mathrm{A}_{i} \mid \mathrm{A}_{j}}\left(\rho_{\mathrm{A}_{i} \mathrm{~A}_{j}}\right) & =\sum_{j=1}^{N} \mathcal{E}\left(\otimes_{s=1}^{s_{j}}\left|\varphi^{s}\right\rangle_{i j}\right) \\
& =\sum_{j=1}^{N} S\left(\rho_{i}^{j}\right)=\sum_{j=1}^{N} \sum_{s=1}^{s_{j}} S\left(\rho_{i ; j}^{s}\right) .
\end{aligned}
$$

Similar to Eqs. (53) and (54), we obtain Eq. (55). Thus, by combining Eqs. (54) and (55), we have

$$
\begin{aligned}
\mathcal{E}_{\mathrm{A}_{i} \mid \overline{\mathrm{A}_{i}}}\left(\rho_{\mathrm{A}_{1} \cdots \mathrm{A}_{n}}\right) & =\sum_{j=1}^{N} \mathcal{E}_{\mathrm{A}_{i} \mid \mathrm{A}_{j}}\left(\rho_{\mathrm{A}_{i} \mathrm{~A}_{j}}\right) \\
& =\sum_{j=1}^{n} \mathcal{E}_{\mathrm{A}_{i} \mid \mathrm{A}_{j}}\left(\rho_{\mathrm{A}_{i} \mathrm{~A}_{j}}\right) .
\end{aligned}
$$


Similar to Eq. (56), we get Eq. (52) for Rényi- $\alpha$ entropy entanglement from its definition in Eq. (5) and the additivity of Rényi- $\alpha$ entropy in Eq. (6). This has completed the proof.

Example 2. Consider a four-partite star quantum network consisting of three EPR states. It can be regarded as a fourpartite pure state on $8 \otimes 2 \otimes 2 \otimes 2$ dimensional Hilbert space, i.e.,

$$
\begin{aligned}
|\phi\rangle_{\mathrm{ABCD}}= & \frac{1}{2 \sqrt{2}}(|0000\rangle+|1001\rangle+|2010\rangle+|3011\rangle \\
& +|4100\rangle+|5101\rangle+|6110\rangle+|7111\rangle)_{\mathrm{ABCD}} .
\end{aligned}
$$

The reduced state of $A$ is given by

$$
\rho_{\mathrm{A}}=\frac{1}{8} \sum_{i=0}^{7}|i\rangle\langle i| .
$$

The EOF of $|\phi\rangle_{\mathrm{A} \mid \mathrm{BCD}}$ is given by $\mathcal{E}\left(|\phi\rangle_{\mathrm{A} \mid \mathrm{BCD}}\right)=3$. For mixed state $\rho_{A B}$, it should take an infimum for the EOF. Note that the reduced density matrix $\rho_{A B}$ of $|\psi\rangle_{A B C D}$ in Eq. (57) has a spectral decomposition

$$
\rho_{\mathrm{AB}}=\frac{1}{4}\left(\left|x_{1}\right\rangle\left\langle x_{1}|+| x_{2}\right\rangle\left\langle x_{2}|+| x_{3}\right\rangle\left\langle x_{3}|+| x_{4}\right\rangle\left\langle x_{4}\right|\right),
$$

where $\left|x_{i}\right\rangle$ are eigenstates given by

$$
\begin{aligned}
& \left|x_{1}\right\rangle_{\mathrm{AB}}=\frac{1}{\sqrt{2}}(|00\rangle+|41\rangle), \\
& \left|x_{2}\right\rangle_{\mathrm{AB}}=\frac{1}{\sqrt{2}}(|10\rangle+|51\rangle), \\
& \left|x_{3}\right\rangle_{\mathrm{AB}}=\frac{1}{\sqrt{2}}(|20\rangle+|61\rangle), \\
& \left.\left|x_{4}\right\rangle_{\mathrm{AB}}=\frac{1}{\sqrt{2}}(|30\rangle+71\rangle\right) .
\end{aligned}
$$

By the Hughston-Jozsa-Wootters (HJW) theorem [53], any pure-state ensemble of $\rho_{A B}$ can be realized as a superposition of $\left|x_{i}\right\rangle$ with $i=1, \ldots, 4$; that is, for arbitrary pure state $|\varphi\rangle_{\mathrm{AB}}=\sum_{i} c_{i}\left|x_{i}\right\rangle$ with $\sum_{i} c_{i}^{2}=1$, its reduced density matrix $\rho_{\mathrm{A}}=\operatorname{Tr}_{\mathrm{B}}\left(|\varphi\rangle_{\mathrm{AB}}\langle\varphi|\right)$ has the same spectrum $\frac{1}{2}$ with double root [54] while all the others are 0 . So, we have $\mathcal{E}\left(|\varphi\rangle_{A B}\right)=1$.

Moreover, the state $\rho_{A B}$ in Eq. (59) can be decomposed into

$$
\rho_{\mathrm{AB}}=\sum_{i} p_{i}\left|\varphi_{i}\right\rangle_{\mathrm{AB}}\left\langle\varphi_{i}\right| .
$$

Thus we have

$$
\mathcal{E}\left(\rho_{\mathrm{AB}}\right)=\sum_{i} p_{i} \mathcal{E}\left(\left|\varphi_{i}\right\rangle_{\mathrm{AB}}\right)=1
$$

from $\mathcal{E}\left(\left|\varphi_{i}\right\rangle_{\mathrm{AB}}\right)=1$ for each state $\left|\varphi_{i}\right\rangle_{\mathrm{AB}}$.

From similar proofs, we show that the EOFs of $\rho_{A C}$ and $\rho_{\mathrm{AD}}$ are

$$
\mathcal{E}\left(\rho_{\mathrm{AC}}\right)=\mathcal{E}\left(\rho_{\mathrm{AD}}\right)=1 .
$$

Consequently, we have

$$
\mathcal{E}\left(|\psi\rangle_{\mathrm{A} \mid \mathrm{BCD}}\right)=\mathcal{E}\left(\rho_{\mathrm{AB}}\right)+\mathcal{E}\left(\rho_{\mathrm{AC}}\right)+\mathcal{E}\left(\rho_{\mathrm{AD}}\right) .
$$

Similarly, for the Rényi- $\alpha$ entanglement measure in Eq. (5), the entanglement distribution satisfies

$$
\begin{gathered}
\mathcal{R}_{\alpha}\left(|\psi\rangle_{\mathrm{A} \mid \mathrm{BCD}}\right)=\mathcal{R}_{\alpha}\left(\rho_{\mathrm{AB}}\right)+\mathcal{R}_{\alpha}\left(\rho_{\mathrm{AC}}\right)+\mathcal{R}_{\alpha}\left(\rho_{\mathrm{AD}}\right) \\
\text { 3. Quantum network with arbitrary multipartite } \\
\text { entangled pure states }
\end{gathered}
$$

\section{Quantum network with arbitrary multipartite entangled pure states}

Generally, it is interesting to show the equality (52) for the multipartite entangled pure state shared by two parties. Consider quantum network $\mathcal{N}_{q_{3}}$, where any two parties $\mathrm{A}_{i}$ and $\mathbf{A}_{j}$ share the states $\tilde{\varrho}_{i j}^{1}, \ldots, \tilde{\varrho}_{i j}^{s}, \sigma_{i j}\left(\varphi_{1}\right), \ldots, \sigma_{i j}\left(\varphi_{t}\right)$, and $\delta_{i j}\left(\vartheta_{1}\right), \ldots, \delta_{i j}\left(\vartheta_{k}\right)$. The joint state shared by any two parties $\mathrm{A}_{i}$ and $\mathrm{A}_{j}$ is given by

$$
\xi_{i j}=\bigotimes_{s=1}^{s_{j}} \widetilde{\varrho}_{i j}^{s} \bigotimes_{t=1}^{t_{j}} \sigma_{i j}\left(\varphi_{t}\right) \bigotimes_{k=1}^{k_{j}} \delta_{i j}\left(\vartheta_{k}\right),
$$

where $\tilde{\varrho}_{i j}^{s}$ denotes the density matrix of the multipartite entangled pure state $|\psi\rangle_{i j}$ with bipartition, $\sigma(\varphi)$ is the density matrix of generalized GHZ state [50] $|\phi(\varphi)\rangle=$ $\cos \varphi|0\rangle^{\otimes m}+\sin \varphi|1\rangle^{\otimes m}$ with any integer $m \geqslant 3$, and $\delta(\vartheta)=$ $\left.\cos ^{2} \vartheta|00\rangle\left\langle 00\left|+\sin ^{2} \vartheta\right| 11\right\rangle\langle 11|\right)$ is the reduced density matrix of any two subsystems obtained by tracing out other subsystems in a generalized GHZ state. Here, $s_{j}, t_{j}$, and $k_{j}$ denote the numbers of $\widetilde{\varrho}, \sigma(\varphi)$, and $\delta(\vartheta)$ shared by two parties $\mathrm{A}_{i}$ and $\mathrm{A}_{j}$.

Thus the total state of $\mathcal{N}_{q_{3}}$ is written as

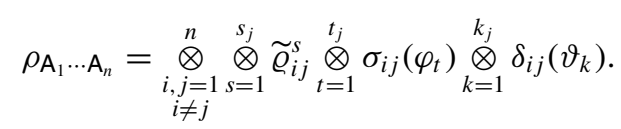

We prove that the monogamy of entanglement measure $\mathcal{Q}$ is valid for $\rho_{\mathrm{A}_{1} \cdots \mathrm{A}_{n}}$ from $\mathcal{N}_{q_{3}}$.

Theorem 4. The entanglement distribution of quantum network $\mathcal{N}_{q_{3}}$ satisfies

$$
\mathcal{Q}_{\mathrm{A}_{i} \mid \overline{\mathrm{A}_{i}}}\left(\rho_{\mathrm{A}_{1} \ldots \mathrm{A}_{n}}\right) \geqslant \sum_{j=1, j \neq i}^{n} \mathcal{Q}_{\mathrm{A}_{i} \mid \mathrm{A}_{j}}\left(\rho_{\mathrm{A}_{i} \mathrm{~A}_{j}}\right),
$$

where $\mathcal{Q}_{\mathrm{A}_{i} \mid \overline{\mathrm{A}_{i}}}$ and $\mathcal{Q}_{\mathrm{A}_{i} \mid \mathrm{A}_{j}}$ are defined in Theorem 3 .

Proof. Suppose that there are $N$ parties $\mathrm{A}_{j}$ who are entangled with the party $A_{i}$. According to Eq. (2), by iteratively using the additivity of von Neumann entropy in Eq. (6), we have

$$
\begin{aligned}
& \mathcal{E}_{\mathrm{A}_{i} \mid \overline{\mathrm{A}_{i}}}\left(\rho_{\mathrm{A}_{1} \cdots \mathrm{A}_{n}}\right)=S\left(\rho_{\mathrm{A}_{i}}\right) \\
& =S\left(\bigotimes_{j=1}^{N}\left(\bigotimes_{s=1}^{s_{j}} \rho_{i ; j}^{s} \bigotimes_{t=1}^{t_{j}} \rho_{i ; j}\left(\varphi_{t}\right) \bigotimes_{k=1}^{k_{j}} \rho_{i ; j}\left(\vartheta_{k}\right)\right)\right) \\
& =\sum_{j=1}^{N} S\left(\bigotimes_{s=1}^{s_{j}} \rho_{i ; j}^{s} \bigotimes_{t=1}^{t_{j}} \rho_{i ; j}\left(\varphi_{t}\right) \bigotimes_{k=1}^{k_{j}} \rho_{i ; j}\left(\vartheta_{k}\right)\right) \\
& =\sum_{j=1}^{N}\left(\sum_{s=1}^{s_{j}} S\left(\rho_{i ; j}^{s}\right)+\sum_{t=1}^{t_{j}} S\left(\rho_{i ; j}\left(\varphi_{t}\right)\right)\right. \\
& \left.+\sum_{k=1}^{k_{j}} S\left(\rho_{i ; j}\left(\vartheta_{k}\right)\right)\right) \text {. }
\end{aligned}
$$


Moreover, it is easy to calculate that

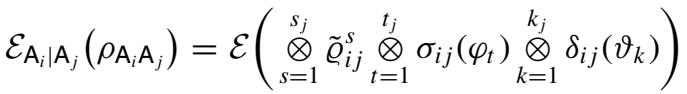

$$
\begin{aligned}
& \leqslant \mathcal{E}\left(\bigotimes_{s=1}^{s_{j}} \tilde{\varrho}_{i j}^{s}\right)+\mathcal{E}\left(\bigotimes_{t=1}^{t_{j}} \sigma_{i j}\left(\varphi_{t}\right)\right) \\
& =S\left(\bigotimes_{s=1}^{s_{j}} \rho_{i ; j}^{s}\right)+S\left(\bigotimes_{t=1}^{t_{j}} \rho_{i ; j}\left(\varphi_{t}\right)\right) \\
& =\sum_{s=1}^{s_{j}} S\left(\rho_{i ; j}^{s}\right)+\sum_{t=1}^{t_{j}} S\left(\rho_{i ; j}\left(\varphi_{t}\right)\right) \text {. }
\end{aligned}
$$

From the additivity of EOF and $\mathcal{E}\left(\otimes_{k=1}^{k_{j}} \delta_{i j}\left(\vartheta_{k}\right)\right)=0$, we get the inequality (69). The equality (70) follows from the definition of EOF in Eq. (2). The equality (71) is obtained by using the additivity of von Neumann entropy in Eq. (3) iteratively. Combining Eqs. (68) and (71), it yields

$$
\begin{aligned}
\mathcal{E}_{\mathrm{A}_{i} \mid \overline{\mathrm{A}_{i}}}\left(\rho_{\mathrm{A}_{1} \cdots \mathrm{A}_{n}}\right) & \geqslant \sum_{j=1, j \neq i}^{N} \mathcal{E}_{\mathrm{A}_{i} \mid \mathrm{A}_{j}}\left(\rho_{\mathrm{A}_{i} \mathrm{~A}_{j}}\right) \\
& =\sum_{j=1, j \neq i}^{n} \mathcal{E}_{\mathrm{A}_{i} \mid \mathrm{A}_{j}}\left(\rho_{\mathrm{A}_{i} \mathrm{~A}_{j}}\right) .
\end{aligned}
$$

Similar to the inequality (72), from Eq. (5) and the additivity of Rényi- $\alpha$ entropy in Eq. (6), it is easy to get the result in Theorem 4.

It also includes entanglement distribution of quantum networks consisting of general entanglement in terms of Tsallis entropy and unified entropy (see Appendix A).

\section{COMMUNICATION CAPACITIES OF QUANTUM NETWORKS}

Quantum networks provide new capabilities for generating and applying quantum entanglement. One fundamental problem is to determine the maximal rate achievable in endto-end transmission of states. This has so far been addressed for single entanglement [1]. Our goal here is to investigate the capacities of general quantum networks $\mathcal{N}_{q}$ consisting of EPR states and GHZ states. One simple method is firstly to transform all GHZ states into EPR states. The analysis that follows is based on the recent method of Ref. [37]. In this case, all the transformed networks should be explored to get the network capacity. This may cost exponential complexity for a large number of GHZ states. Instead, the present method implies a one-shot scheme for characterizing the network capacity of the hybrid network. The present method has always polynomial complexity.

For any bipartite entanglement $|\varphi\rangle_{A B}$, it can be regarded as a quantum channel with the capacity [1] $\mathcal{C}_{\mathrm{AB}}=S\left(\rho_{\mathrm{A}}\right)=$ $\mathcal{E}_{\mathrm{A} \mid \mathrm{B}}\left(|\varphi\rangle_{\mathrm{AB}}\right)$, where $S\left(\rho_{\mathrm{A}}\right)$ is the von Neumann entropy of party $A$. As a result, the total capacity of quantum channel $\left\{\xi_{i j}\right\}$ connecting the parties $A_{i}$ and $A_{j}$ in quantum network $\mathcal{N}_{q}$ is given by

$$
\begin{aligned}
S\left(\rho_{i}^{j}\right) & =\mathcal{E}_{\mathrm{A}_{i} \mid \mathrm{A}_{j}}\left(\rho_{\mathrm{A}_{i} \mathrm{~A}_{j}}\right) \\
& =n_{1}+n_{2},
\end{aligned}
$$

where $n_{1}$ and $n_{2}$ denote the respective number of EPR states and GHZ states shared by parties $\mathrm{A}_{i}$ and $\mathrm{A}_{j}$ and $S\left(\rho_{i}^{j}\right)$ represents the von Neumann entropy of the reduced density matrix $\rho_{i}^{j}$ by tracing out the system of $j$, i.e., $\rho_{i}^{j}=\operatorname{Tr}_{j}\left(\left|\xi_{i j}\right\rangle\left\langle\xi_{i j}\right|\right)$. This equality allows us to evaluate the capacity of quantum networks via von Neumann entropy. It is noteworthy that Rényi entropy is also available for evaluating channel capacity.

Similar to classical network theory [55], consider an $n$ partite entangled quantum network $\mathcal{N}_{q}(\mathcal{A}, \Omega)$. Let $\mathbf{s}$ and $\mathbf{t}$ be the source and sink, respectively. Define the flow $f_{i \rightarrow j}$ in a quantum network $\mathcal{N}_{q}$ as the number of qubits which are reliably transmitted from $A_{i}$ to $A_{j}$ along the channel $\xi_{i j}$; that is,

$$
f_{i \rightarrow j} \geqslant 0 \text {. }
$$

For the quantum channel consisting of EPR states [49] and GHZ states [50], the capacity between $A_{i}$ and $A_{j}$ is defined in Eq. (73), which means the maximal number of qubits transmitted. Inspired by the method in Ref. [37], the flow is bounded by the two-way quantum capacity of the associated channel $\xi_{i j}$, i.e.,

$$
f_{i \rightarrow j} \leqslant \mathcal{C}\left(\xi_{i j}\right)=S\left(\rho_{i}^{j}\right),
$$

where $S\left(\rho_{i}^{j}\right)$ has the formation of Eq. (73). In addition, at any intermediate party, the number of qubits simultaneously received must be equal to the number of qubits simultaneously transmitted through all the party-to-party communications with neighbor parties. This means that for any $i \in \mathcal{A} \backslash\{\mathbf{s}, \mathbf{t}\}$, we have

$$
\sum_{j \in \mathcal{A}} f_{i \rightarrow j}=0 .
$$

This does not hold for the source $\mathbf{s}$ and the sink $\mathbf{t}$, for which we impose

$$
\sum_{j \in \mathcal{A}} f_{\mathbf{s} \rightarrow j}=-\sum_{j \in \mathcal{A}} f_{\mathbf{t} \rightarrow j}=f,
$$

where $f$ is known as the value of the flow. This is an achievable end-to-end rate since it represents the total number of qubits which are transmitted by the source and correspondingly received by the sink via all of the end-to-end communication. Thus, analogous to a classical network, we define a flow in a quantum network as follows.

Definition 1. A flow of a quantum network $\mathcal{N}_{q}(\mathcal{A}, \Omega)$ from $\mathbf{s}$ to $\mathbf{t}$ is given by a function $f: \Omega \longrightarrow \mathbb{R}^{+}$, such that $f$ satisfies the following.

(i) In the capacity constraint, the flow is bounded by the capacity of the channel; that is, for any $\xi_{i j} \in \Omega, f_{i \rightarrow j}$ satisfies

$$
f_{i \rightarrow j} \leqslant \mathcal{C}\left(\xi_{i j}\right) \text {. }
$$

(ii) In the conservation of flow, the flow leaving the party $j$ is equal to the flow entering the party $j$; that is,

$$
\sum_{\left\{i: \xi_{i j} \in \Omega\right\}} f_{i \rightarrow j}=\sum_{\left\{k: \xi_{j k} \in \Omega\right\}} f_{j \rightarrow k},
$$

for any party $j \in \mathcal{A}$, other than the sender (source) $\mathbf{s}$ and receiver (sink) $\mathbf{t}$. 
Herein, $f_{i \rightarrow j}$ denotes the number of qubits transmitted from party $i$ to party $j$ by the quantum channel $\xi_{i j}$. If $f_{i \rightarrow j}=\mathcal{C}\left(\xi_{i j}\right)$, then the quantum channel $\xi_{i j}$ is saturated. Otherwise, $\xi_{i j}$ is unsaturated.

Definition 2. A maximum flow $\operatorname{MF}(\mathcal{N})$ of quantum network $\mathcal{N}_{q}(\mathcal{A}, \Omega)$ is the maximum amount of flow $f$ from the source $\mathbf{s}$ to the sink $\mathbf{t}$; that is,

$$
f_{\max }=\max \{f\} .
$$

To address the maximal flow of given network $\mathcal{N}_{q}(\mathcal{A}, \Omega)$, the main idea is to find the cut of associated undirected graph $\mathcal{G}=(\mathcal{V}, \Omega)$, where $\mathcal{V}$ consists of all vertices and $\Omega$ consists of all edges. Here, one vertex schematically denotes one party. One edge $(i, j)$ connecting two vertices schematically denotes an EPR state shared by the corresponding parties $\mathrm{A}_{i}$ and $\mathrm{A}_{j}$. The GHZ state shared by $m$ parties is associated with the hyperedge connecting $m$ vertices. This correspondence is reasonable because of the equality $S\left(\rho_{\mathrm{A}_{i}}\right)=S\left(\rho_{\mathrm{A}_{j}}\right)$ for any GHZ state $\rho_{\mathrm{A}_{1} \ldots \mathrm{A}_{m}}, 1 \leqslant i<j \leqslant m$. Thus the quantum network $\mathcal{N}_{q}$ is associated with an undirected finite hypergraph $\mathcal{G}$. With this correspondence, the weight of each edge $(i, j)$ or hyperedge is the unit, i.e., the entanglement entropy of the EPR state or GHZ state. We then adopt the standard definition of a cut set for a graph [55]. A cut $T_{\text {cut }}$ of $\mathcal{G}$ is a bipartition $\left(\mathbf{S}_{1}, \mathbf{S}_{2}\right)$ of $\mathcal{V}$ such that $\mathbf{s} \in \mathbf{S}_{1}$ and $\mathbf{t} \in \mathbf{S}_{2}$. The cut set $\widetilde{T}_{\text {cut }}$ of $T_{\text {cut }}$ is the set of edges so that the removal of these edges disconnects the source $\mathbf{s}$ and the sink $\mathbf{t}$.

Definition 3. An edge cut set $\widetilde{T}_{\text {cut }}$ consists of edges such that there exists a bipartition $\left(\mathbf{S}_{1}, \mathbf{S}_{2}\right)$ of all the vertices in $\mathcal{V}$ satisfying $\mathbf{s} \in \mathbf{S}_{1}$ and $\mathbf{t} \in \mathbf{S}_{2}$; that is, $\widetilde{T}_{\text {cut }}=\{(i, j): i \in$ $\left.\mathbf{S}_{1}, j \in \mathbf{S}_{2}\right\}$. Then the capacity of an edge cut set $T_{\text {cut }}$ is given by

$$
\mathcal{C}\left(\mathbf{S}_{1}, \mathbf{S}_{2}\right)=\sum_{(i, j) \in \widetilde{T}_{\text {cut }}} \mathcal{C}(i, j),
$$

where $\mathcal{C}(i, j)$ denotes the weight of edge $(i, j)$.

Definition 4. If $\mathcal{C}\left(T_{\text {cut }}\right) \geqslant \mathcal{C}\left(T_{\text {cut }}^{\prime}\right)$ holds for any cut $T_{\text {cut }}$ in graph $\mathcal{G}$, then $T_{\text {cut }}^{\prime}$ is a minimal cut of $\mathcal{G}$; that is, a minimum cut is a cut with the minimum capacity, with the capacity given by

$$
\mathcal{C}\left(T_{\text {cut }}^{\prime}\right)=\min _{T_{\text {cut }}} \mathcal{C}\left(\mathbf{S}_{1}, \mathbf{S}_{2}\right)
$$

Significantly, the flow in a classical network obeys the celebrated max-flow min-cut theorem [55]. We prove a similar result for quantum settings.

Theorem 5. For a given quantum network $\mathcal{N}_{q}$ associated with undirected graph $\mathcal{G}$, the maximal flow $f_{\max }$ is equal to the minimal cut of $\mathcal{G}$; that is,

$$
f_{\max }=\min _{T_{\text {cut }}} \mathcal{C}\left(\mathbf{S}_{1}, \mathbf{S}_{2}\right) .
$$

In what follows, we prove Theorem 5. Firstly, we resort to following Lemma 1 in a classical network [56,57].

Lemma 1. Let $f$ be a flow on a network $\mathcal{N}$ and $T_{\text {cut }}$ be a cut $T_{\text {cut }}=\left(\mathbf{S}_{1}, \mathbf{S}_{2}\right)$ with the capacity $\mathcal{C}\left(T_{\text {cut }}\right)$. Then the flow satisfies

$$
f \leqslant \mathcal{C}\left(T_{\text {cut }}\right)
$$

where the equality holds if and only if each edge $(i, j)$ in the cut $T_{\text {cut }}=\left(\mathbf{S}_{1}, \mathbf{S}_{2}\right)$ is saturated, i.e., $f_{i \rightarrow j}=\mathcal{C}(i, j)$, and each edge $(j, i)$ in $T_{\text {cut }}=\left(\mathbf{S}_{2}, \mathbf{S}_{1}\right)$ has zero flow with $f_{j \rightarrow i}=0$.

The proof of Lemma 1 is presented in Appendix B.

Proof of Theorem 5. Now, consider a quantum network $\mathcal{N}_{q}(\mathcal{A}, \Omega)$ consisting of quantum channels defined as EPR states and GHZ states. There exists a cut $T_{\text {cut }}$ in $\widetilde{T}_{\text {cut }}$ of the associated graph $\mathcal{G}$ separating $\mathbf{S}_{1}$ and $\mathbf{S}_{2}$. Similar to Lemma 1, we get

$$
f\left(\mathbf{S}_{1}, \mathbf{S}_{2}\right) \leqslant \mathcal{C}\left(T_{\text {cut }}\right),
$$

where the equality holds if and only if each channel $\xi_{i j}$ in the cut $T_{\text {cut }}=\left(\mathbf{S}_{1}, \mathbf{S}_{2}\right)$ is saturated, and $f_{i \rightarrow j}=\mathcal{C}\left(\xi_{i j}\right)=\mathcal{C}(i, j)$. As is shown in Refs. [1,58], one can perfectly transmit one qubit by using the quantum channel of the EPR state [49] or GHZ state [50]. Thereby, it is feasible to achieve $f_{i \rightarrow j}=$ $\mathcal{C}(i, j)$ from the point of view of quantum communication. Note that the transmission of information in quantum communication is one way, i.e., $f\left(\mathbf{S}_{2}, \mathbf{S}_{1}\right)=0$. Furthermore, we get the following result.

Lemma 2. If a flow $f$ and cut $T_{\text {cut }}=\left(\mathbf{S}_{1}, \mathbf{S}_{2}\right)$ on a quantum network $\mathcal{N}_{q}(\mathcal{A}, \Omega)$ satisfy $f=\mathcal{C}\left(T_{\text {cut }}\right)$, then $f$ is a maximum flow and the cut $T_{\text {cut }}$ is a minimum cut.

Note that any flow $f^{\prime}$ of the network $\mathcal{N}_{q}$ satisfies $f^{\prime} \leqslant$ $\mathcal{C}\left(T_{\text {cut }}\right)=f$. On the other hand, for any cut $T_{\text {cut }}^{\prime}$ of the associated graph $\mathcal{G}$, it follows that $\mathcal{C}^{\prime}\left(T_{\text {cut }}\right) \geqslant f=\mathcal{C}\left(T_{\text {cut }}\right)$. Thus $f$ is the maximum flow, and the cut $T_{\text {cut }}$ is the minimum cut. As a result, we obtain the claim in Theorem 5 for the quantum network $\mathcal{N}_{q}(\mathcal{A}, \Omega)$. This means that the maximal flow of $\mathcal{N}_{q}(\mathcal{A}, \Omega)$ is equal to the minimum-capacity cut of the associated graph $\mathcal{G}$. This completes the proof of Theorem 5.

Theorem 5 states that the maximum flow from $\mathbf{s}$ to $\mathbf{t}$ in quantum network $\mathcal{N}_{q}$ is equal to the minimum cut that separates $\mathbf{s}$ and $\mathbf{t}$ in the corresponding graph $\mathcal{G}$. This provides a general method similar to the point-to-point quantum protocol [1] for general quantum networks beyond the recent result [37]. In applications, for a given quantum network $\mathcal{N}_{q}$ consisting of EPR states and GHZ states under local unitary operations, the network capacity can be evaluated by extending the Ford-Fulkerson algorithm [59] as shown in Algorithm 1.

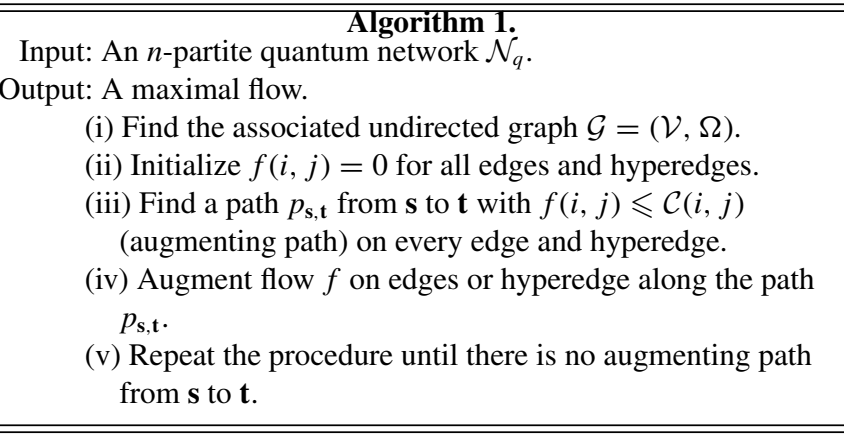

Example 3. Consider the maximization of the flow from $\mathbf{s}$ to $\mathbf{t}$ as shown in Fig. 5(a) by applying the extended FordFulkerson algorithm. It is easy to check that the maximum flow from $\mathbf{s}$ to $\mathbf{t}$ is equal to 7 , which is consistent with the minimum capacity of all source-sink cuts in a corresponding 


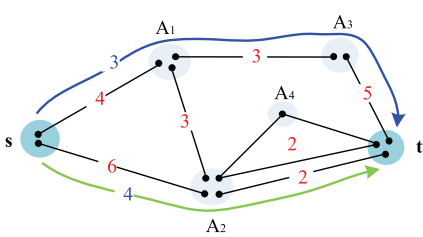

(a)

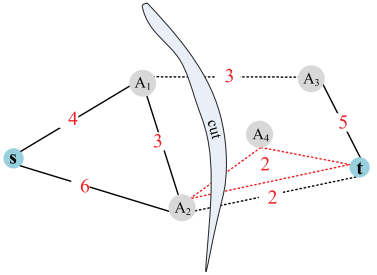

(b)
FIG. 5. (a) Maximum flow of six-partite quantum network $\mathcal{N}_{q}$. $\mathbf{s}$ is the source, while $\mathbf{t}$ is the sink. Three parties, $A_{2}, A_{4}$, and $\mathbf{t}$, are connected by two GHZ states, and the remaining parties are connected by multiple EPR states. The red integer on a quantum channel denotes its capacity, i.e., the number of EPR states or GHZ states. The blue and green arrows are two maximal flows. (b) Minimum cut of the associated graph of $\mathcal{N}_{q}$. The EPR state is denoted as one edge, while the GHZ state is denoted as one hyperedge (red lines). A source-sink cut achieves the minimum cut of 7 .

undirected graph as shown in Fig. 5(b). The iterations of Example 3 are given in Appendix C.

\section{NETWORK TOPOLOGY CLASSIFICATION}

Actual quantum networks have a complex topology which significantly affects the communication efficiency. It is natural to consider how to identify the network configuration for a given network. One method is to explore the measurement statistics by using the covariance matrix $[38,39]$ or the generalized Finner inequality [60] in a device-independent manner. The other is from coherence theory [61]. These methods can be applied to characterize quantum networks assisted by specific measurement.

\section{A. Classifying networks based on the von Neumann entropy}

In this section, our goal is to consider a beyond problem of classifying configurations of a given set of networks with lower complexity. It is well known that two local unitary equivalent multipartite entangled states have the same entanglement entropy with respect to any bipartition. We show that the converse holds for quantum networks consisting of EPR states and GHZ states. Especially, for a given $n$-partite quantum network $\mathcal{N}_{q}$, denote the characteristic vector of quantum network $\mathcal{N}_{q}$ as

$$
S_{\mathcal{N}_{q}}=\left(S\left(\rho_{\mathrm{A}_{1}}\right), S\left(\rho_{\mathrm{A}_{2}}\right), \ldots, S\left(\rho_{\mathrm{A}_{n}}\right)\right),
$$

where $S\left(\rho_{\mathrm{A}_{i}}\right)$ denotes the von Neumann entropy of the reduced state of party $\mathrm{A}_{i}$ of $\mathcal{N}_{q}$. We present a configuration classification under the local unitary equivalence by virtue of the characteristic vector of quantum networks.

Theorem 6. Assume $\mathcal{N}_{1}$ and $\mathcal{N}_{2}$ are two quantum networks consisting of EPR states and multipartite GHZ states, where any two parties $A_{i}$ and $A_{j}$ in each network share no more than one entanglement. Then, $\mathcal{N}_{1}$ and $\mathcal{N}_{2}$ have unitary equivalence if and only if their characteristic vectors are equal to each other, i.e., $S_{\mathcal{N}_{1}}=S_{\mathcal{N}_{2}}$.

Proof. Consider the following two cases.

(i) Case 1: $n=3$. In this case, there are three types of quantum networks, $\mathcal{N}_{1}, \mathcal{N}_{2}$, and $\mathcal{N}_{3}$, as shown in Fig. 6. The

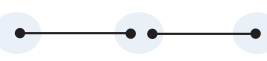

(a)

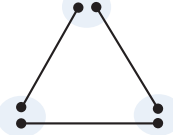

(b)

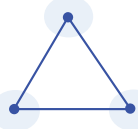

(c)
FIG. 6. Three types of tripartite quantum network, $\mathcal{N}_{1}, \mathcal{N}_{2}$, and $\mathcal{N}_{3}$, consisting of EPR states or GHZ states, where any two parties share no more than an entanglement. (a) A chain quantum network consisting of two EPR states. (b) A triangle quantum network consisting of three EPR states. (c) A triangle quantum network consisting of one GHZ state.

vectors of von Neumann entropy of quantum networks are given by

$$
\begin{aligned}
& S_{\mathcal{N}_{1}}=(1,2,1), \\
& S_{\mathcal{N}_{2}}=(2,2,2), \\
& S_{\mathcal{N}_{3}}=(1,1,1) .
\end{aligned}
$$

It follows that the characteristic vectors of von Neumann entropy for tripartite quantum networks consisting of EPR states or GHZ states are different from each other; that is, they are unique for each kind of tripartite quantum network. Note that the von Neumann entropy is invariant under the local unitary operations. This has proved the result.

(ii) Case 2: $n>3$. Assume that the result holds for $n \leqslant$ $k-1$; that is, for any pair of quantum networks $\mathcal{N}_{1}$ and $\mathcal{N}_{2}$ there exist local unitary operations $U_{j}$ such that

$$
\otimes_{j=1}^{n} U_{j} \rho_{\mathcal{N}_{1}} U_{j}^{\dagger}=\rho_{\mathcal{N}_{2}}
$$

if $S_{\mathcal{N}_{1}}=S_{\mathcal{N}_{2}}$, where $S_{\mathcal{N}_{j}}$ denotes the characteristic vector defined by von Neumann entropies for quantum network $\mathcal{N}_{j}$ consisting of EPR states or GHZ states. Here, each party in the networks may perform local unitary operations. This means that the quantum network consisting of EPR states and GHZ states is unique under local unitary operations.

In what follows, we will prove the result for quantum networks $\mathcal{N}_{3}$ with $n=k$. In fact, this will be proved by two subcases. The main idea is that the new network $\mathcal{N}_{3}$ can be regarded as an extended network of $\mathcal{N}_{1}$.

Assume that $\mathcal{N}_{3}$ consists of $k$ parties $\mathrm{A}_{1}, \mathrm{~A}_{2}, \ldots, \mathrm{A}_{k}$ who share EPR states and GHZ states, as shown in Fig. 7(a). The quantum network $\mathcal{N}_{3}$ will be decomposed into two subnetworks $\mathcal{N}_{3 ; 1}$ as shown in Fig. 7(b) and $\mathcal{N}_{3 ; 2}$ as shown in Fig. 7(c), where $\mathcal{N}_{3 ; 1}$ consists of $k-1$ parties $\left(\mathrm{A}_{2}, \ldots, \mathrm{A}_{k}\right.$, for example) of $\mathcal{N}_{3}$, and $\mathcal{N}_{3 ; 2}$, which has at most $k-1$ parties, consists of at least one party $\mathrm{A}_{1}$ and some parties $\mathrm{A}_{s_{1}}, \ldots, \mathbf{A}_{s_{m}}$ entangled with $A_{1}$ of $\mathcal{N}_{3}$.

Consider the subnetwork $\mathcal{N}_{3 ; 1}$ with $k-1$ parties. The characteristic vector of $\mathcal{N}_{3 ; 1}$ is given by $S_{\mathcal{N}_{3 ; 1}}$. For the subnetwork $\mathcal{N}_{3 ; 2}$, the characteristic vector is given by $S_{\mathcal{N}_{3,2}}$. These networks consist of EPR states and GHZ states. Moreover, the local von Neumann entropy of each party satisfies the additivity, namely,

$$
S_{j}=m_{\mathrm{EPR}}^{(j)}+m_{\mathrm{GHZ}}^{(j)},
$$

where $m_{\mathrm{EPR}}$ and $m_{\mathrm{GHZ}}$ denote the respective number of EPR states and GHZ states shared by the party $\mathrm{A}_{j}$. Note that the 


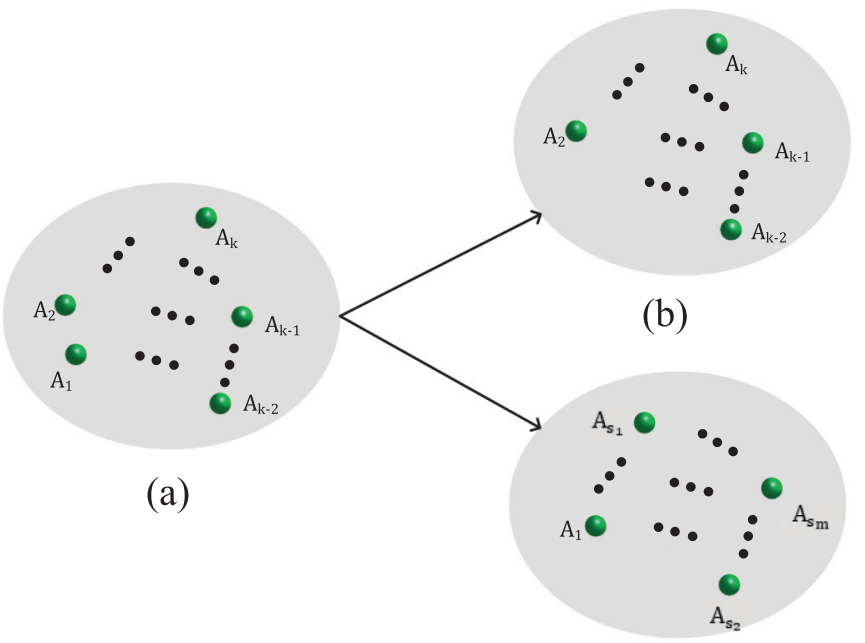

(c)

FIG. 7. Schematic quantum network $\mathcal{N}_{3}$ decomposed into two subnetworks, $\mathcal{N}_{3 ; 1}$ and $\mathcal{N}_{3 ; 2}$. (a) $\mathcal{N}_{3}$ consisting of $\mathrm{A}_{1}, \mathrm{~A}_{2}, \ldots, \mathrm{A}_{k-1}, \mathrm{~A}_{k}$. (b) Subnetwork $\mathcal{N}_{3 ; 1}$ consisting of $k-1$ parties $\left(A_{2}, \ldots, A_{k}\right.$, for example) of $\mathcal{N}_{1}$ and the party $A_{k}$. (c) $\mathcal{N}_{3 ; 2}$ consisting of at least one party $A_{1}$ and some parties $A_{s_{1}}, \ldots, A_{s_{m}}$ entangled with $\mathrm{A}_{1}$ of $\mathcal{N}_{3}$.

von Neumann entropy is invariant under the local unitary operations. It follows that the characteristic vector of quantum networks consisting of EPR and GHZ states satisfies the additivity; that is,

$$
S_{\mathcal{N}_{3}}=S_{\mathcal{N}_{3 ; 1}}+S_{\mathcal{N}_{3 ; 2}} .
$$

Moreover, $\mathcal{N}_{3 ; 1}$ and $\mathcal{N}_{3 ; 2}$ have at most $k-1$ parties. Hence we get that the new network generated by combinations of the subnetworks $\mathcal{N}_{3 ; 1}$ and $\mathcal{N}_{3 ; 2}$ is equivalent to the network $\mathcal{N}_{3}$. From the assumption, it follows that the characteristic vectors of $\mathcal{N}_{3 ; 1}$ and $\mathcal{N}_{3 ; 2}$ are unique under the local unitary operations. This means that for each of the other networks $\mathcal{N}_{3 ; 1}^{\prime}$ and $\mathcal{N}_{3 ; 2}^{\prime}$ consisting of EPR and GHZ states we have

$$
\otimes_{j} U_{j} \rho_{\mathcal{N}_{3 ; s}} U_{j}^{\dagger}=\rho_{\mathcal{N}_{3 ; s}^{\prime}}, \quad s=1,2,
$$

if $S_{\mathcal{N}_{3 ; s}}=S_{\mathcal{N}_{3 ; s}^{\prime}}$. This means that the characteristic vectors of $\mathcal{N}_{3 ; s}$ and $\mathcal{N}_{3 ; s}^{\prime}$ are equal to each other, where $U_{j}$ is the unitary operation. This implies that

$$
S_{\mathcal{N}_{3}}=S_{\mathcal{N}_{3}^{\prime}},
$$

where

$$
S_{\mathcal{N}_{3}^{\prime}}=S_{\mathcal{N}_{3 ; 1}^{\prime}}+S_{\mathcal{N}_{3 ; 2}^{\prime}} \cdot
$$

Note Eq. (91) means that there exist local unitary operations satisfying

$$
\otimes_{j} U_{j} \rho_{\mathcal{N}_{3}} U_{j}^{\dagger}=\rho_{\mathcal{N}_{3}^{\prime}} .
$$

So, $\mathcal{N}_{3}$ is local unitary (LU) equivalent to the network $\mathcal{N}_{3}^{\prime}$; that is, the characteristic vector of quantum network $\mathcal{N}_{3}$ is unique. This completes the proofs of Theorem 6.

According to Theorem 6, we present a quantum network classification method by virtue of the characteristic vector of

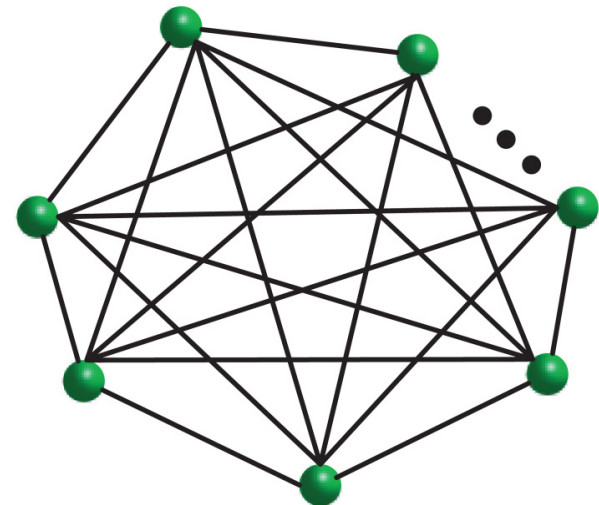

FIG. 8. A multipartite complete graphlike network consisting of EPR states.

von Neumann entropy. We present some examples to explain the main idea.

Example 4. Consider a simple network with $n$ parties $A_{1}, A_{2}, \ldots, A_{n}$. Here, adjacent parties share no more than one EPR pair as shown in Fig. 2(a) for a chain quantum network, Fig. 2(b) for a cyclic network, and Fig. 2(c) for a star quantum network. The von Neumann entropy vectors of these networks are given by $(1,2, \ldots, 2,1),(2, \ldots, 2)$, and $(n-1,1, \ldots, 1)$, respectively, which are different. This shows that these networks are inequivalent to each other under local unitary operations.

Example 5. Consider an $n$-partite complete graphlike network $\mathcal{N}_{\mathbf{A}}$, as shown in Fig. 8. Any pair of two parties shares an EPR state. Each party shares $n-1$ EPR states with others. There are $n(n-1) / 2$ EPR states. The characteristic vector of $\mathcal{N}_{\mathbf{A}}$ is given by $(n-1, n-1, \ldots, n-1)$, which is different from that for the networks in Fig. 2.

Theorem 6 provides a simple method to classify the given set of networks consisting of EPR states and GHZ states. In application, it only requires one to evaluate $n$ number of von Neumann entropies $S\left(\rho_{\mathrm{A}_{i}}\right)$, which has linear complexity beyond the exponential complexity for evaluating all entanglement entropies of $S\left(\rho_{\mathrm{A}_{i_{1}} \ldots \mathrm{A}_{i_{t}}}\right), 1 \leqslant i_{1}<\cdots<i_{t} \leqslant n$. The drawback is from the assumption of no more than one entanglement shared by a given group of parties. Otherwise, two counterexamples are shown in Fig. 9. From Fig. 9(a), the triangle network consisting of three EPR states is inequivalent to its consisting of two GHZ states even for the same characteristic vector $(2,2,2)$. The other counterexample is cyclic networks consisting of four EPR states or two GHZ states and one EPR state in Fig. 9(b) with characteristic vector $(2,2,2,2)$. These can be extended for any cyclic quantum network. Thus a new method beyond the characteristic vector of von Neumann entropy should be explored to verify these cyclic quantum networks. One possibility is from Shannon mutual information [62] derived from local projection measurements.

\section{B. Classifying networks based on the mutual information}

In this section, we classify quantum networks based on the mutual information. We firstly recall the definition of mutual information. 

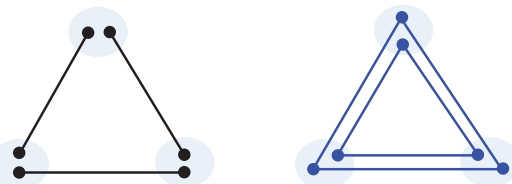

(a)
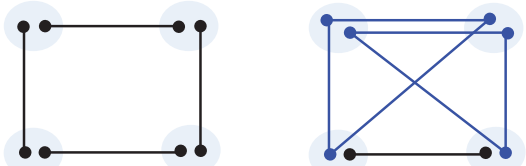

(b)

FIG. 9. Different networks with the same characteristic vector. (a) Triangle network consisting of three EPR states (black lines) or two GHZ states (blue lines). (b) Four-partite network consisting of four EPR states or two GHZ states and one EPR state.

Shannon mutual information [62] describes how much information can be determined about a random variable $X$, by knowing the value of a correlated random variable $\mathrm{Y}$. The Shannon mutual information for a tripartite system can be written as

$$
\begin{aligned}
I(\mathrm{X}: \mathrm{Y}: \mathrm{Z})= & H(\mathrm{XYZ})+H(\mathrm{X})+H(\mathrm{Y})+H(\mathrm{Z}) \\
& -H(\mathrm{XY})-H(\mathrm{YZ})-H(\mathrm{XZ}),
\end{aligned}
$$

where $H(\mathrm{X})=-\sum_{\mathrm{x} \in \mathrm{X}} p(\mathrm{x}) \log _{2} p(\mathrm{x})$ is the entropy of $\mathrm{X}$ and $H(\mathrm{XY})=-\sum_{\mathrm{x} \in \mathrm{X}, \mathrm{y} \in \mathrm{Y}} p(\mathrm{X}, \mathrm{y}) \log _{2} p(\mathrm{X}, \mathrm{y})$ is the joint entropy of $\mathrm{X}$ and $\mathrm{Y} ; p(\mathrm{X}, \mathrm{Y})$ is the joint probability distribution of $\mathrm{X}$ and $\mathrm{Y}$.

Lemma 3. The two types of cyclic networks in Figs. 9(a) and 9 (b) can be verified by Shannon mutual information derived from local projection measurements.

The proof of Lemma 3 is given in Appendix D. From Lemma 3, we obtain the following theorem.

Theorem 7. For any quantum network $\mathcal{N}_{\mathrm{A}}$ consisting of EPR states contains some cyclic subnetworks, there is another quantum network $\mathcal{N}_{\mathrm{B}}$ with the same characteristic vector of von Neumann entropy as $\mathcal{N}_{\mathrm{A}}$ such that they have different network topologies under local operations.

Proof. As is illustrated in Fig. 10(a), quantum network $\mathcal{N}_{\mathrm{A}}$ contains four subnetworks, $\mathcal{N}_{1}, \mathcal{N}_{2}, \mathcal{N}_{3}$, and $\mathcal{N}_{4}$. Here, threepartite subnetwork $\mathcal{N}_{1}$ has parties $A_{1}, A_{3}$, and $A_{5} . \mathcal{N}_{2}$ contains parties $\mathrm{A}_{2}, \mathrm{~A}_{4}$, and $\mathrm{A}_{6} . \mathcal{N}_{3}$ contains parties $\mathrm{A}_{n-2}, \mathrm{~A}_{n-1}$, and $A_{n}$. All of these subnetworks consist of three EPR states. A four-partite cyclic subnetwork $\mathcal{N}_{4}$ consists of parties $A_{1}, A_{2}$, $A_{3}$, and $A_{4}$, who share an EPR state with adjacent parties. By replacing EPR states with GHZ states, we can construct another quantum network $\mathcal{N}_{\mathrm{B}}$ as shown in Fig. 10(b), where the subnetwork $\mathcal{N}_{i}$ is transformed into the corresponding subnetwork $\mathcal{N}^{\prime}{ }_{i}, i=1, \ldots, 4$.

From Lemma 3, it is easy to show that the subnetworks $\mathcal{N}_{i}$ are inequivalent to the subnetworks $\mathcal{N}^{\prime}{ }_{i}$. This follows that two networks $\mathcal{N}_{\mathrm{A}}$ and $\mathcal{N}_{\mathrm{B}}$ are inequivalent even if they have the same characteristic vector of von Neumann entropies as $S\left(\rho_{\mathrm{A}_{i}}\right)=S\left(\rho_{\mathrm{B}_{i}}\right)=(4,4,5,5,2,2, \ldots, 3,3,2)$. Thus, for any quantum network containing a triangle network or four-partite cyclic subnetwork consisting of EPR states, there is another

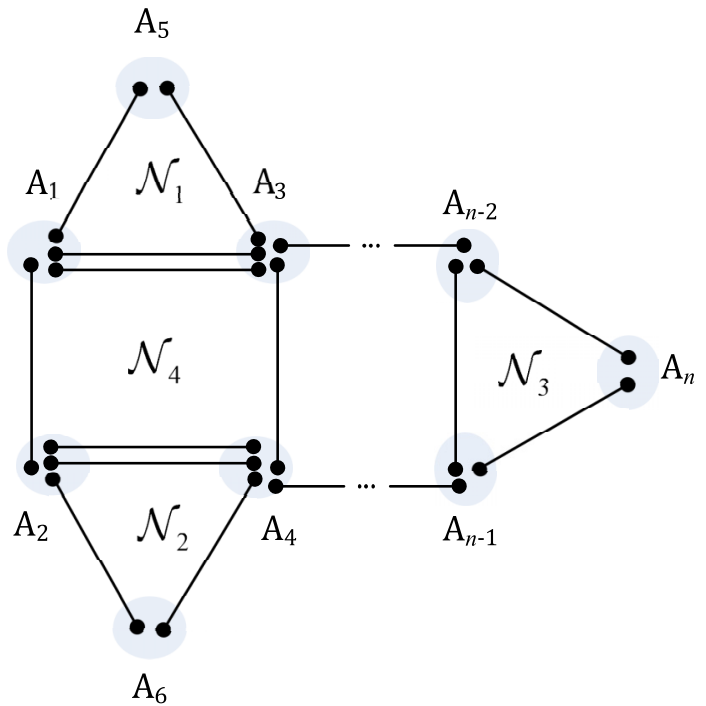

(a)

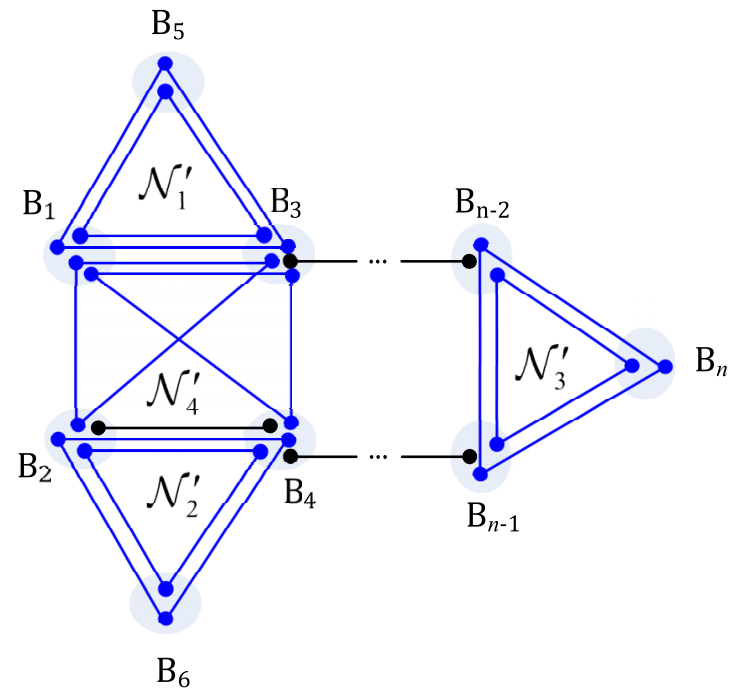

(b)

FIG. 10. Inequivalent networks with the same characteristic vector defined by von Neumann entropies. (a) Quantum network $\mathcal{N}_{\mathrm{A}}$ consisting of EPR states. (b) Quantum network $\mathcal{N}_{\mathrm{B}}$ consisting of EPR states and GHZ states.

quantum network with the same characteristic vector of von Neumann entropies such that they are inequivalent under local unitary operations. This completes the proof.

Theorem 7 shows that the characteristic vector of von Neumann entropies cannot be used for classifying all quantum networks. Fortunately, this problem can be resolved by using Lemma 3.

\section{DISCUSSION AND CONCLUSION}

Quantum entropy provides an easy way to characterize the entanglement distribution of specific quantum networks. This strong monogamy relation implies interesting applications in 
quantum communications and quantum network configurations. The present method is useful for generic settings of a quantum network consisting of EPR states and GHZ states. These kinds of networks are universal for measurement-based quantum computation [63]. A natural problem is for general entangled networks. Specially, it should be useful for exploring quantum networks consisting of mixed states, which may bring to light new features going beyond pure systems. Another is for the maximum-flow problem on noisy networks including dephasing and erasure channels.

To sum up, we have proved the strong monogamy relation for quantum networks consisting of any bipartite entangled pure states and generalized GHZ states. These relations provide the feature of high-dimensional entanglement beyond qubit states. This implies the celebrated max-flow min-cut theorem on quantum networks based on von Neumann entropy. Finally, we presented a network classification using Shannon or von Neumann entropies of the measurement statistics. This provides a device-independent manner to verify the quantum network configuration under local unitary operations. The present results are interesting in quantum entanglement, quantum networks, and quantum information processing.

\section{ACKNOWLEDGMENTS}

We are thankful for the help of S. Fei, Y. Guo, X. Ding, and $X$. Chen. This work was supported by the National Natural Science Foundation of China (Grants No. 61772437 and No. 62172341), and Shenzhen Institute for Quantum Science and Engineering, Southern University of Science and Technology (Grant No. SIQSE202105).

\section{APPENDIX A: EXTENSION OF THEOREM 1 WITH OTHER ENTROPIES}

In this Appendix, we investigate the entanglement distribution of quantum network $\mathcal{N}_{q_{2}}(\mathcal{A}, \xi)$ in terms of Tsallis- $q$ entanglement and unified $(q, s)$-entropy entanglement.

\section{Tsallis- $q$ entropy}

Theorem 8. Assume that a quantum network $\mathcal{N}_{q_{2}}(\mathcal{A}, \xi)$ is connected by arbitrary multipartite entangled pure states.

(i) For $0<q<1$, the entanglement distribution of $\mathcal{N}_{q_{2}}$ satisfies

$$
\mathcal{T}_{\mathrm{A}_{i} \mid \overline{\mathrm{A}_{i}}}^{(q)}\left(\rho_{\mathrm{A}_{1} \ldots \mathrm{A}_{n}}\right) \geqslant \sum_{j=1, j \neq i}^{n} \mathcal{T}_{\mathrm{A}_{i} \mid \mathrm{A}_{j}}^{(q)}\left(\rho_{\mathrm{A}_{i} \mathrm{~A}_{j}}\right) .
$$

(ii) For $q>1$, the entanglement distribution of $\mathcal{N}_{q_{2}}$ satisfies

$$
\mathcal{T}_{\mathrm{A}_{i} \mid \overline{\mathrm{A}_{i}}}^{(q)}\left(\rho_{\mathrm{A}_{1} \cdots \mathrm{A}_{n}}\right) \geqslant \sum_{j=1, j \neq i}^{n} \mathcal{T}_{\mathrm{A}_{i} \mid \mathrm{A}_{j}}^{(q)}\left(\rho_{\mathrm{A}_{i} \mathrm{~A}_{j}}\right),
$$

where $\mathcal{T}_{\mathrm{X} \mid \mathrm{Y}}^{(q)}$ is the Tsallis- $q$ entanglement in Eq. (8) with respect to the bipartition $\mathrm{X}$ and $\mathrm{Y}$, and $\overline{\mathrm{A}_{i}}$ denotes all parties except for $\mathrm{A}_{i}$.

Proof. Suppose that there exist $N$ parties $\mathrm{A}_{j}$ who are entangled with the party $\mathrm{A}_{i}$. From Eq. (8), for the case $0<q<1$, we have

$$
\begin{aligned}
\mathcal{T}_{\mathrm{A}_{i} \mid \overline{\mathrm{A}}_{i}}^{(q)}\left(\rho_{\mathrm{A}_{1} \ldots \mathrm{A}_{n}}\right) & =S_{q}\left(\rho_{\mathrm{A}_{i}}\right) \\
& =S_{q}\left(\bigotimes_{j=1}^{N}\left(\bigotimes_{s=1}^{s_{j}} \rho_{i ; j}^{s}\right)\right) \\
& \leqslant \sum_{j=1}^{N} S_{q}\left(\bigotimes_{s=1}^{s_{j}} \rho_{i ; j}^{s}\right),
\end{aligned}
$$

where the inequality (A3) is derived from using the additivity of the Tsallis- $q$ entropy in Eq. (10) iteratively, $\rho_{i ; j}^{s}$ is the reduced density matrix of the subsystems owned by party $\mathrm{A}_{i}$ by tracing out the subsystems owned by party $\mathrm{A}_{j}$ in a multipartite entangled pure state $\left|\psi^{s}\right\rangle$, i.e., $\rho_{i ; j}^{s}=\operatorname{Tr}_{j}\left(\left|\psi^{s}\right\rangle_{i j}\left\langle\psi^{s}\right|\right)$.

In addition, we have

$$
\begin{aligned}
\sum_{j=1}^{N} \mathcal{T}_{\mathrm{A}_{i} \mid \overline{\mathrm{A}_{i}}}^{(q)}\left(\rho_{\mathrm{A}_{i} \mathrm{~A}_{j}}\right) & =\sum_{j=1}^{N} \mathcal{T}^{(q)}\left(\bigotimes_{s=1}^{s_{j}}\left|\psi^{s}\right\rangle_{i j}\right) \\
& =\sum_{j=1}^{N} S_{q}\left(\rho_{i}^{j}\right) \\
& =\sum_{j=1}^{N} S_{q}\left(\underset{\left.\bigotimes_{s=1}^{s_{j}} \rho_{i ; j}^{s}\right) .}{ }\right.
\end{aligned}
$$

Here, the equality (A4) follows from the definition of Tsallis- $q$ entanglement in Eq. (8), and the equality (A5) is due to $\rho_{i}^{j}=$ $\otimes_{s=1}^{s_{j}} \rho_{i, j}^{s}$, i.e., all the states of $\rho_{i ; j}^{s}$, s are not entangled. The inequality (A3) and equality (A5) yield to the inequality (A1).

Similarly, we obtain the inequality (A2) for $q>1$. This completes the proof.

\section{Unified $(q, s)$ entropy}

Similar to Theorem 8, we obtain the following theorem.

Theorem 9. Assume that a quantum network $\mathcal{N}_{q_{2}}(\mathcal{A}, \xi)$ consists of arbitrary multipartite entangled pure states.

(i) For $q \geqslant 1$ and $s \geqslant 0$, the entanglement distribution of $\mathcal{N}_{q_{2}}$ satisfies

$$
\mathcal{U}_{\mathrm{A}_{i} \mid \mathrm{A}_{i}}^{(q, s)}\left(\rho_{\mathrm{A}_{1} \cdots \mathrm{A}_{n}}\right) \geqslant \sum_{j=1, j \neq i}^{n} \mathcal{U}_{\mathrm{A}_{i} \mid \mathrm{A}_{j}}^{(q, s)}\left(\rho_{\mathrm{A}_{i} \mathrm{~A}_{j}}\right) .
$$

(ii) For $0<q<1$ and $s>0$, the entanglement distribution of $\mathcal{N}_{q_{2}}$ satisfies

$$
\mathcal{U}_{\mathrm{A}_{i} \mid \mathrm{A}_{i}}^{(q, s)}\left(\rho_{\mathrm{A}_{1} \ldots \mathrm{A}_{n}}\right) \geqslant \sum_{j=1, j \neq i}^{n} \mathcal{U}_{\mathrm{A}_{i} \mid \mathrm{A}_{j}}^{(q, s)}\left(\rho_{\mathrm{A}_{i} \mathrm{~A}_{j}}\right),
$$

where $\mathcal{U}_{\mathrm{X} \mid \mathrm{Y}}^{(q, s)}$ is the unified $(q, s)$-entropy entanglement in Eq. (13) with respect to the bipartition $X$ and $Y$, and $\overline{A_{i}}$ denotes all parties except for $A_{i}$. 
Proof. The proof is similar to that for Theorem 8. Similar to the inequality (A3), from Eq. (13) and the subadditivity of the unified $(q, s)$-entropy, for $q \geqslant 1$ and $s \geqslant 0$, we get

$$
\begin{aligned}
\mathcal{U}_{\mathrm{A}_{i} \mid \overline{\mathrm{A}_{i}}}^{(q, s)}\left(\rho_{\mathrm{A}_{1} \ldots \mathrm{A}_{n}}\right) & =S_{q, s}\left(\rho_{\mathrm{A}_{i}}\right) \\
& =S_{q, s}\left(\stackrel{\bigotimes}{j=1}_{j}^{N}\left(\stackrel{s}{s=1}_{s}^{s_{j}} \rho_{i ; j}^{s}\right)\right) \\
& \leqslant \sum_{j=1}^{N} S_{q, s}\left(\stackrel{s}{s=1}_{s}^{s_{j}} \rho_{i ; j}^{s}\right) .
\end{aligned}
$$

Similar to Eq. (A5), from Eq. (13) we have

$$
\mathcal{U}_{\mathrm{A}_{i} \mid \mathrm{A}_{j}}^{(q, s)}\left(\rho_{\mathrm{A}_{i} \mathrm{~A}_{j}}\right)=\sum_{j=1}^{N} S_{q, s}\left(\stackrel{s_{j}}{\otimes} \rho_{s=1}^{s} \rho_{i ; j}^{s}\right) .
$$

Thus Eqs. (A9) and (A10) imply the inequality (A6) for $q \geqslant 1$ and $s \geqslant 0$.

Similarly, we can obtain the inequality (A7) for $0<q<1$ and $s>0$. This completes the proof.

\section{APPENDIX B: PROOF OF LEMMA 1}

Proof. Denote $f=f\left(\mathbf{S}_{1}, \mathbf{S}_{2}\right)-f\left(\mathbf{S}_{2}, \mathbf{S}_{1}\right)$. On the one hand, the flow conservation law implies that $f\left(\mathbf{S}_{1}, \mathbf{S}_{2}\right) \leqslant$ $\mathcal{C}\left(T_{\text {cut }}\right)$ and $f\left(\mathbf{S}_{2}, \mathbf{S}_{1}\right) \geqslant 0$. Note that $f=f\left(\mathbf{S}_{1}, \mathbf{S}_{2}\right)-$ $f\left(\mathbf{S}_{2}, \mathbf{S}_{1}\right) \leqslant \mathcal{C}\left(T_{\text {cut }}\right)$, i.e.,

$$
f \leqslant \mathcal{C}\left(T_{\text {cut }}\right) \text {. }
$$

For $f\left(\mathbf{S}_{1}, \mathbf{S}_{2}\right) \leqslant \mathcal{C}\left(T_{\text {cut }}\right)$, the equality holds if and only if each edge $(i, j)$ in cut $T_{\text {cut }}=\left(\mathbf{S}_{1}, \mathbf{S}_{2}\right)$ is saturated. Besides, $f\left(\mathbf{S}_{2}, \mathbf{S}_{1}\right) \geqslant 0$, where the equality holds if and only if each edge $(j, i)$ in $\left(\mathbf{S}_{2}, \mathbf{S}_{1}\right)$ has zero flow. So, $f=\mathcal{C}\left(T_{\text {cut }}\right)$ holds if and only if each edge $(i, j)$ in the cut $T_{\text {cut }}=$ $\left(\mathbf{S}_{1}, \mathbf{S}_{2}\right)$ is saturated and each edge $(j, i)$ in $\left(\mathbf{S}_{2}, \mathbf{S}_{1}\right)$ has zero flow.

\section{APPENDIX C: THE ITERATION OF EXAMPLE 3}

In this Appendix, embarking on presenting the iteration of Example 3, we recall the following lemma.

Lemma 4 (Ref. [57]). Let $f$ be a flow on a classical network $\mathcal{N}$. Then $f$ is the maximum flow only if $f$ has no augmenting paths.

This lemma is also available for a quantum network $\mathcal{N}_{q}$ consisting of EPR states and GHZ states. Hence the main idea for the maximal flow of quantum network $\mathcal{N}_{q}$ is to find augmenting paths. We apply the Ford-Fulkerson algorithm [57] that sends the maximal possible amount of flow at each iteration; that is, the flow equals the capacity of the path under consideration, where $f_{i}$ denotes the amount of flow sent in iteration $i$.

Iteration 1 . We find a path $s \rightarrow 1 \rightarrow 2 \rightarrow t$ that can carry a positive flow; then the maximum flow we can send along this path is $f_{1}=\min \{4,3,2\}=2$, as shown in Fig. 11(a). In Fig. 11(b), we obtain a residual network with updated link capacities resulting from pushing the flow along the path.

Iteration 2. Similarly, we find an augmenting path $s \rightarrow$ $1 \rightarrow\{2,4\} \rightarrow t$ to send a positive flow. The maximum flow

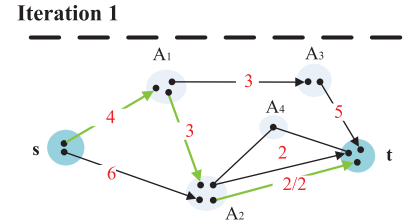

(a)
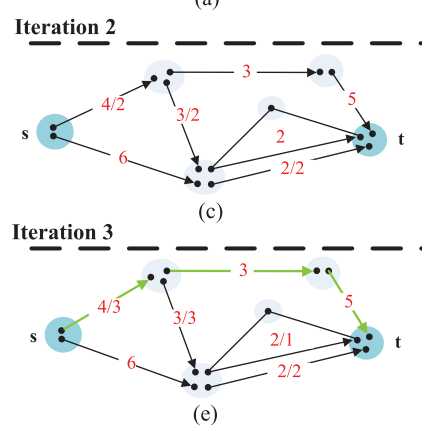

(e)

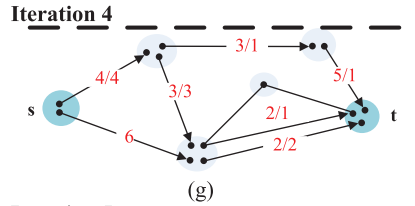

(g)

Iteration 5

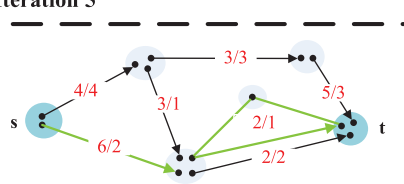

(i)

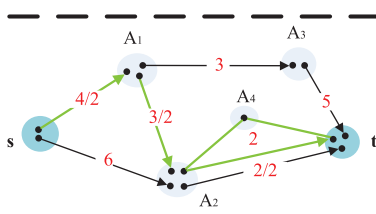

(b)

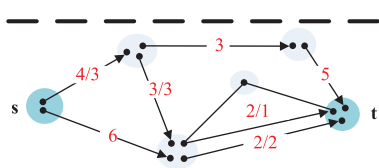

(d)

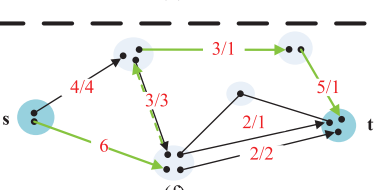

(f)

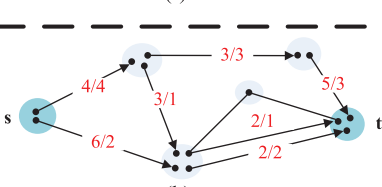

(h)

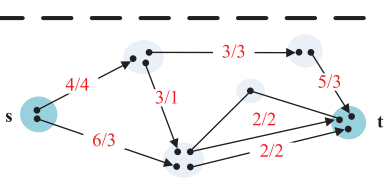

(j)
FIG. 11. The iterations for finding the maximum flow in Example 2. (a) The augmenting path $s \rightarrow 1 \rightarrow 2 \rightarrow t$. (b) The residual network in the first iteration by adding the maximum flow $f_{1}$. (c) The augmenting path $s \rightarrow 1 \rightarrow\{2,4\} \rightarrow t$. (d) The residual network in the second iteration by adding a maximum flow $f_{2}$. (e) The augmenting path $s \rightarrow 1 \rightarrow 3 \rightarrow t$. (f) The residual network in the third iteration by adding a maximum flow $f_{3}$. $(\mathrm{g})$ The augmenting path $s \rightarrow 2 \rightarrow 1 \rightarrow 3 \rightarrow t$. (h) The residual network in the fourth iteration by adding a maximum flow $f_{4}$. (i) The augmenting path $s \rightarrow\{2,4\} \rightarrow t$. (j) The residual network in the fifth iteration by adding a maximum flow $f_{5}$.

along this path is then given by $f_{2}=\min \{4-2,3-2,2\}=$ 1 , as shown in Fig. 11(c). We get a residual network with updated capacities shown in Fig. 11(d).

Iteration 3. We find a path $s \rightarrow 1 \rightarrow 3 \rightarrow t$ which sends a maximum flow along this path given by $f_{3}=\min \{4-$ $3,3,5\}=1$, as shown in Fig. 11(e). A residual network is updated as shown in Fig. 11(f).

Iteration 4. For the path $s \rightarrow 2 \rightarrow 1 \rightarrow 3 \rightarrow t$, a maximum flow along this path is given by $f_{4}=\min \{6,3,3-$ $1,5-1\}=2$ as shown in Fig. $11(\mathrm{~g})$. A residual network with updated capacities resulting from pushing the flow along the path is given by Fig. 11(h).

Iteration 5. We find a path $s \rightarrow\{2,4\} \rightarrow t$ for sending a positive flow. The maximum flow we can send along this path is given by $f_{5}=\min \{6-2,2-1\}=1$, as shown in Fig. 11(i). We obtain a residual network with updated link capacities resulting from pushing the flow along the path, as shown in Fig. 11(j). 


\section{APPENDIX D: PROOF OF LEMMA 3}

Proof. The quantum networks $\mathcal{N}_{1}$ and $\mathcal{N}_{2}$ shown in Fig. 9(a) are inequivalent under local unitary operations. The total state of the system for network $\mathcal{N}_{1}$ is given by

$$
\begin{aligned}
\left|\mu^{1}\right\rangle= & \frac{1}{\sqrt{2}}(|00\rangle+|11\rangle)_{\mathrm{A}_{1} \mathrm{~B}_{1}} \otimes \frac{1}{\sqrt{2}}(|00\rangle+|11\rangle)_{\mathrm{A}_{1} \mathrm{C}_{1}} \\
& \otimes \frac{1}{\sqrt{2}}(|00\rangle+|11\rangle)_{\mathrm{B}_{1} \mathrm{C}_{1}},
\end{aligned}
$$

which may be written as

$$
\begin{aligned}
\left|\mu^{1}\right\rangle= & \frac{1}{2 \sqrt{2}}(|000\rangle+|201\rangle+|012\rangle+|213\rangle \\
& +|120\rangle+|321\rangle+|132\rangle+|333\rangle)_{A_{1} B_{1} C_{1}},
\end{aligned}
$$

where we regard the local two-qubit systems as a fourdimensional system with the computation basis $\{|0\rangle, \ldots,|4\rangle\}$. After projection measurements are performed on quantum state $\left|\mu^{1}\right\rangle$ in Eq. (D2), we get a joint probability distribution

$$
p\left(x_{1} x_{2} x_{3}\right)=\frac{1}{8}
$$

where $x_{1} x_{2} x_{3} \in\{000,201,012,213,120,321,132,333\}$.

For the network $\mathcal{N}_{2}$ as shown in Fig. 9(b) the total state is given by

$$
\left|\mu^{2}\right\rangle=\frac{1}{\sqrt{2}}(|000\rangle+|111\rangle)_{\mathrm{A}_{1} \mathrm{~B}_{1} \mathrm{C}_{1}}^{\otimes 2}
$$

that is,

$$
\left|\mu^{2}\right\rangle=\frac{1}{\sqrt{2}}(|000\rangle+|111\rangle+|222\rangle+|333\rangle)_{A_{1} B_{1} C_{1}} .
$$

For quantum state $\left|\mu^{2}\right\rangle$ in Eq. (D5), after projection measurements are performed, a joint probability distribution is obtained as

$$
p\left(y_{1} y_{2} y_{3}\right)=\frac{1}{4}
$$

where $y_{1} y_{2} y_{3} \in\{000,111,222,333\}$.

From Eq. (95), it is easy to check that the Shannon mutual information of subsystems in $\mathcal{N}_{1}$ and $\mathcal{N}_{2}$ is given by

$$
\begin{aligned}
& I\left(\mathrm{~A}_{1}: \mathrm{B}_{1}: \mathrm{C}_{1}\right)=3, \\
& I\left(\mathrm{~A}_{2}: \mathrm{B}_{2}: \mathrm{C}_{2}\right)=2 .
\end{aligned}
$$

Similarly, the Shannon mutual information of subsystems in $\mathcal{N}_{3}$ and $\mathcal{N}_{4}$ in Fig. 9 (b) is given by

$$
\begin{aligned}
& I\left(\mathrm{~A}_{3}: \mathrm{B}_{3}: \mathrm{C}_{3}: \mathrm{D}_{3}\right)=4, \\
& I\left(\mathrm{~A}_{4}: \mathrm{B}_{4}: \mathrm{C}_{4}: \mathrm{D}_{4}\right)=3,
\end{aligned}
$$

which is different from that in Eqs. (D7) and (D8). This means that the Shannon mutual information as a character can be used to distinguish two networks.

Note that the von Neumann entropy is unitary invariant. Another interesting method for distinguishing network topologies is to use the von Neumann entropy of the extended system by copying the original quantum state into two copies. Specially, by introducing an auxiliary qubit in the state $|0\rangle$ locally, each party performs a controlled-NOT operation on local systems, where the original qubit is the controlling qubit while the auxiliary qubit is the target. The total system $\left|\mu^{1}\right\rangle$ in Eq. (D1) is then changed into

$$
\begin{aligned}
\left|v^{1}\right\rangle= & \frac{1}{\sqrt{2}}\left(|0\rangle^{\otimes 4}+|1\rangle^{\otimes 4}\right)_{\mathrm{A}_{1} \mathrm{a}_{1} \mathrm{~B}_{1} \mathrm{~b}_{1}} \\
& \otimes \frac{1}{\sqrt{2}}\left(|0\rangle^{\otimes 4}+|1\rangle^{\otimes 4}\right)_{\mathrm{A}_{1} \mathrm{a}_{1} \mathrm{C}_{1} \mathrm{c}_{1}} \\
& \otimes \frac{1}{\sqrt{2}}\left(|0\rangle^{\otimes 4}+|1\rangle^{\otimes 4}\right)_{\mathrm{B}_{1} \mathrm{~b}_{1} \mathrm{C}_{1} \mathrm{c}_{1} .}
\end{aligned}
$$

Similarly, for the network $\mathcal{N}_{2}$ in Fig. 9(b), the state $\left|\mu^{2}\right\rangle$ in Eq. (D4) is changed into

$$
\begin{aligned}
\left|v^{2}\right\rangle= & \frac{1}{\sqrt{2}}\left(|0\rangle^{\otimes 6}+|1\rangle^{\otimes 6}\right)_{\mathrm{A}_{2} \mathrm{a}_{2} \mathrm{~B}_{2} \mathrm{~b}_{2} \mathrm{C}_{2} \mathrm{c}_{2}} \\
& \otimes \frac{1}{\sqrt{2}}\left(|0\rangle^{\otimes 6}+|1\rangle^{\otimes 6}\right)_{\mathrm{A}_{2} \mathrm{a}_{2} \mathrm{~B}_{2} \mathrm{~b}_{2} \mathrm{C}_{2} \mathrm{C}_{2} .}
\end{aligned}
$$

For quantum states in Eqs. (D11) and (D12), we obtain

$$
\begin{aligned}
& S\left(\rho_{\mathrm{A}_{1} \mathrm{~B}_{1} \mathrm{C}_{1}}\right)=3, \\
& S\left(\rho_{\mathrm{A}_{2} \mathrm{~B}_{2} \mathrm{C}_{2}}\right)=2,
\end{aligned}
$$

which are different from each other, where $S(\mathrm{X})$ is the von Neumann entropy of the subsystem $X$. This means that we can identify two different quantum networks by using the von Neumann entropy for the system $\mathrm{A}_{i} \mathrm{~B}_{i} \mathrm{C}_{i}$ with $i=1,2$.

Similarly, the von Neumann entropies of the subsystems $\mathrm{A}_{i}, \mathrm{~B}_{i}, \mathrm{C}_{i}$, and $\mathrm{D}_{i}(i=3,4)$ for $\mathcal{N}_{3}$ and $\mathcal{N}_{4}$ are given by

$$
\begin{aligned}
& S\left(\rho_{\mathrm{A}_{3} \mathrm{~B}_{3} \mathrm{C}_{3} \mathrm{D}_{3}}\right)=4, \\
& S\left(\rho_{\mathrm{A}_{4} \mathrm{~B}_{4} \mathrm{C}_{4} \mathrm{D}_{4}}\right)=3,
\end{aligned}
$$

respectively, which elucidates two inequivalent networks $\mathcal{N}_{3}$ and $\mathcal{N}_{4}$ under local unitary operations. This provides another method for classifying quantum networks.
[1] C. H. Bennett, G. Brassard, C. Crépeau, R. Jozsa, A. Peres, and W. K. Wootters, Teleporting an Unknown Quantum State via Dual Classical and Einstein-Podolsky-Rosen Channels, Phys. Rev. Lett. 70, 1895 (1993).

[2] C. H. Bennett and S. J. Wiesner, Communication via Oneand Two-Particle Operators on Einstein-Podolsky-Rosen States, Phys. Rev. Lett. 69, 2881 (1992).
[3] M. Hillery, V. Bužek, and A. Berthiaume, Quantum secret sharing, Phys. Rev. A 59, 1829 (1999).

[4] N. Gisin, G. Ribordy, W. Tittel, and H. Zbinden, Quantum cryptography, Rev. Mod. Phys. 74, 145 (2002).

[5] R. Horodecki, P. Horodecki, M. Horodecki, and K. Horodecki, Quantum entanglement, Rev. Mod. Phys. 81, 865 (2009). 
[6] A. K. Ekert, Quantum Cryptography Based on Bell's Theorem, Phys. Rev. Lett. 67, 661 (1991).

[7] J. Eisert, M. Cramer, and M. B. Plenio, Colloquium: Area laws for the entanglement entropy, Rev. Mod. Phys. 82, 277 (2010).

[8] L. Amico, R. Fazio, A. Osterloh, and V. Vedral, Entanglement in many-body systems, Rev. Mod. Phys. 80, 517 (2008).

[9] J. P. Dowling and G. J. Milburn, Quantum technology: the second quantum revolution, Philos. Trans. R. Soc. A 361, 1655 (2003).

[10] B. Terhal, Is entanglement monogamous? IBM J. Res. Dev. 48, 71 (2004).

[11] V. Coffman, J. Kundu, and W. K. Wootters, Distributed entanglement, Phys. Rev. A 61, 052306 (2000).

[12] T. J. Osborne and F. Verstraete, General Monogamy Inequality for Bipartite Qubit Entanglement, Phys. Rev. Lett. 96, 220503 (2006).

[13] T. R. de Oliveira, M. F. Cornelio, and F. F. Fanchini, Monogamy of entanglement of formation, Phys. Rev. A 89, 034303 (2014).

[14] Y. K. Bai, Y. F. Xu, and Z. D. Wang, General Monogamy Relation for the Entanglement of Formation in Multiqubit Systems, Phys. Rev. Lett. 113, 100503 (2014).

[15] Y. Guo and G. Gour, Monogamy of the entanglement of formation, Phys. Rev. A 99, 042305 (2019).

[16] X. N. Zhu and S. M. Fei, Entanglement monogamy relations of qubit systems, Phys. Rev. A 90, 024304 (2014).

[17] Z. X. Jin and S. M. Fei, Monogamy relations of all quantum correlation measures for multipartite quantum systems, Opt. Commun. 446, 39 (2019).

[18] Y. Luo, T. Tian, L. H. Shao, and Y. M. Li, General monogamy of Tsallis $q$-entropy entanglement in multiqubit systems, Phys. Rev. A 93, 062340 (2016).

[19] J. S. Kim and B. C. Sanders, Monogamy of multi-qubit entanglement using Rényi entropy, J. Phys. A: Math. Theor. 43, 445305 (2010).

[20] J. S. Kim and B. C. Sanders, Unified entropy, entanglement measures and monogamy of multi-party entanglement, J. Phys. A: Math. Theor. 44, 295303 (2011).

[21] Y. K. Bai, Y. F. Xu, and Z. D. Wang, Hierarchical monogamy relations for the squared entanglement of formation in multipartite systems, Phys. Rev. A 90, 062343 (2014).

[22] W. Song, Y. K. Bai, M. Yang, and Z. L. Cao, General monogamy relation of multi-qubit system in terms of squared Rényi- $\alpha$ entanglement, Phys. Rev. A 93, 022306 (2016).

[23] C. Lancien, S. Di Martino, M. Huber, M. Piani, G. Adesso, and A. Winter, Should Entanglement Measures be Monogamous or Faithful? Phys. Rev. Lett. 117, 060501 (2016).

[24] A. Khan, J. ur Rehman, K. Wang, and H. Shin, Unified monogamy relations of multipartite entanglement, Sci. Rep. 9, 16419 (2019).

[25] M. Koashi and A. Winter, Monogamy of quantum entanglement and other correlations, Phys. Rev. A 69, 022309 (2004).

[26] X. Yang and M. X. Luo, Unified monogamy relation of entanglement measures, Quantum Inf. Process. 20, 108 (2021).

[27] Y.-C. Ou and H. Fan, Monogamy inequality in terms of negativity for three-qubit states, Phys. Rev. A 75, 062308 (2007).

[28] M.-X. Luo and S.-M. Fei, Robust multipartite entanglement without entanglement breaking, Phys. Rev. Research 3, 043120 (2021).
[29] M. Żukowski, A. Zeilinger, M. A. Horne, and A. K. Ekert, "Event-Ready-Detectors" Bell Experiment Via Entanglement Swapping, Phys. Rev. Lett. 71, 4287 (1993).

[30] L. M. Duan, M. D. Lukin, J. I. Cirac, and P. Zoller, Longdistance quantum communication with atomic ensembles and linear optics, Nature (London) 414, 413 (2001).

[31] J. Kimble, The quantum Internet, Nature (London) 453, 1023 (2008).

[32] S. Wehner, D. Elkouss, and R. Hanson, Quantum internet: A vision for the road ahead, Science 362, 9288 (2018).

[33] J. Cirac, A. K. Ekert, S. F. Huelga, and C. Macchiavello, Distributed quantum computation over noisy channels, Phys. Rev. A 59, 4249 (1999).

[34] A. Broadbent, J. Fitzsimons, and E. Kashefi, Universal blind quantum computation, in 200950 th Annual IEEE Symposium on Foundations of Computer Science (IEEE, Atlanta, GA, 2009), pp. 517-526.

[35] D. Gottesman, T. Jennewein, and S. Croke, Longer-Baseline Telescopes Using Quantum Repeaters, Phys. Rev. Lett. 109, 070503 (2012)

[36] P. Komar, E. M. Kessler, M. Bishof, L. Jiang, A. S. Sørensen, J. Ye, and M. D. Lukin, A quantum network of clocks, Nat. Phys. 10, 582 (2014).

[37] S. Pirandola, End-to-end capacities of a quantum communication network, Commun. Phys. 2, 51 (2019).

[38] A. Kela, K. Von Prillwitz, J. Aberg, R. Chaves, and D. Gross, Semidefinite tests for latent causal structures, IEEE Trans. Inf. Theory 66, 339 (2020).

[39] J. Aberg, R. Nery, C. Duarte, and R. Chaves, Semidefinite Tests for Quantum Network Topologies, Phys. Rev. Lett. 125, 110505 (2020).

[40] C. H. Bennett, D. P. DiVincenzo, J. A. Smolin, and W. K. Wootters, Mixed-state entanglement and quantum error correction, Phys. Rev. A 54, 3824 (1996).

[41] M. A. Nielsen and I. L. Chuang, Quantum Computation and Quantum Information (Cambridge University Press, Cambridge, 2000).

[42] W. van Dam and P. Hayden, Rényi-entropic bounds on quantum communication, arXiv:quant-ph/0204093.

[43] J. S. Kim, Tsallis entropy and entanglement constraints in multiqubit systems, Phys. Rev. A 81, 062328 (2010).

[44] G. A. Raggio, Properties of $q$-entropies, J. Math. Phys. (Melville, NY) 36, 4785 (1995).

[45] P. N. Rathie and I. J. Taneja, Unified $(r, s)$-entropy and its bivariate measures, Inf. Sci. 54, 23 (1991).

[46] R. Horodecki, P. Horodecki, and M. Horodecki, Quantum $\alpha$ entropy inequalities: independent condition for local realism? Phys. Lett. A 210, 377 (1996).

[47] C. Tsallis, Possible generalization of Boltzmann-Gibbs statistics, J. Stat. Phys. 52, 479 (1988).

[48] X. Hu and Z. Ye, Generalized quantum entropy, J. Math. Phys. (Melville, NY) 47, 023502 (2006).

[49] A. Einstein, B. Podolsky, and N. Rosen, Can Quantum Mechanical Description of Physical Reality Be Considered Complete? Phys. Rev. 47, 777 (1935).

[50] D. M. Greenberger, M. A. Horne, and A. Zeilinger, Going beyond Bell's theorem, in Bell's Theorem, Quantum Theory and Conceptions of the Universe, edited by M. Kafatos (Kluwer, Dordrecht, 1989), pp. 69-72. 
[51] W. Dür, G. Vidal, and J. I. Cirac, Three qubits can be entangled in two inequivalent ways, Phys. Rev. A 62, 062314 (2000).

[52] W. K. Wootters, Entanglement of Formation of an Arbitrary State of Two Qubits, Phys. Rev. Lett. 80, 2245 (1998).

[53] L. P. Hughston, R. Jozsa, and W. K. Wootters, A complete classification of quantum ensembles having a given density matrix, Phys. Lett. A 183, 14 (1993).

[54] G. Vidal and R. F. Werner, Computable measure of entanglement, Phys. Rev. A 65, 032314 (2002).

[55] V. I. Voloshin, Introduction to Graph and Hypergraph Theory (Nova Science, Hauppauge, NY, 2009).

[56] P. Elias, A. Feinstein, and C. E. Shannon, A note on the maximum flow through a network, IEEE Trans. Inf. Theory 2, 117 (1956).
[57] L. R. Ford and D. R. Fulkerson, Maximal flow through a network, Can. J. Math. 8, 399 (1956).

[58] A. Karlsson and M. Bourennane, Quantum teleportation using three-particle entanglement, Phys. Rev. A 58, 4394 (1998).

[59] T. H. Cormen, C. E. Leiserson, R. L. Rivest, and C. Stein, Introduction to Algorithms, 2nd ed. (MIT Press, Cambridge, MA, 2001).

[60] M. X. Luo, Network configuration theory for all networks, arXiv:2107.05846.

[61] T. Kraft, C. Spee, X. D. Yu, and O. Gühne, Characterizing quantum networks: Insights from coherence theory, Phys. Rev. A 103, 052405 (2021)

[62] C. E. Shannon and W. Weaver, The Mathematical Theory of Communication (University of Illinois Press, Urbana, 1998).

[63] R. Raussendorf and H. J. Briegel, A One-Way Quantum Computer, Phys. Rev. Lett. 86, 5188 (2001). 